\title{
Universiteit
}

Leiden

The Netherlands

\section{Further evidence for chemical fractionation from ultraviolet observations of carbon monoxide}

Federman, S.R.; Lambert, D.L.; Sheer, Y.; Cardelli, J.A.; Andersson, B.G.; Dishoeck, E.F. van; Zsargo, J.

\section{Citation}

Federman, S. R., Lambert, D. L., Sheer, Y., Cardelli, J. A., Andersson, B. G., Dishoeck, E. F. van, \& Zsargo, J. (2003). Further evidence for chemical fractionation from ultraviolet observations of carbon monoxide. Astrophys. J., 591, 986-999. Retrieved from https://hdl.handle.net/1887/2190

Version: $\quad$ Not Applicable (or Unknown)

License:

Downloaded from: https://hdl.handle.net/1887/2190

Note: To cite this publication please use the final published version (if applicable). 


\title{
FURTHER EVIDENCE FOR CHEMICAL FRACTIONATION FROM ULTRAVIOLET OBSERVATIONS OF CARBON MONOXIDE ${ }^{1}$
}

\author{
S. R. Federman, ${ }^{2,3}$ David L. Lambert, ${ }^{4}$ Yaron Sheffer, ${ }^{2,4}$ Jason A. Cardelli, ${ }^{5,6}$ B.-G. Andersson, ${ }^{2,7,8}$ \\ EWINE F. van Dishoeck, ${ }^{9}$ AND J. Zsargó ${ }^{2,8}$ \\ Received 2001 August 7; accepted 2003 March 22
}

\begin{abstract}
Ultraviolet absorption from interstellar ${ }^{12} \mathrm{CO}$ and ${ }^{13} \mathrm{CO}$ was detected toward $\rho$ Oph $\mathrm{A}$ and $\chi \mathrm{Oph}$. The measurements were obtained at medium resolution with the Goddard High Resolution Spectrograph on the Hubble Space Telescope. Column density ratios, $N\left({ }^{12} \mathrm{CO}\right) / N\left({ }^{13} \mathrm{CO}\right)$, of $125 \pm 23$ and $117 \pm 35$ were derived for the sight lines toward $\rho$ Oph A and $\chi$ Oph, respectively. A value of $1100 \pm 600$ for the ratio $N\left({ }^{12} \mathrm{C}^{16} \mathrm{O}\right) /$ $N\left({ }^{12} \mathrm{C}^{18} \mathrm{O}\right)$ toward $\rho$ Oph A was also obtained. Absorption from vibrationally excited $\mathrm{H}_{2}\left(v^{\prime \prime}=3\right)$ was clearly seen toward this star as well. The ratios are larger than the isotopic ratios for carbon and oxygen appropriate for ambient interstellar material. Since for both carbon and oxygen the more abundant isotopomer is enhanced, selective isotopic photodissociation plays the key role in the fractionation process for these directions. The enhancement arises because the more abundant isotopomer has lines that are more optically thick, resulting in more self-shielding from dissociating radiation. A simple argument involving the amount of self-shielding [from $N\left({ }^{12} \mathrm{CO}\right)$ ] and the strength of the ultraviolet radiation field permeating the gas (from the amount of vibrationally excited $\mathrm{H}_{2}$ ) shows that selective isotopic photodissociation controls the fractionation seen in these two sight lines, as well as the sight line to $\zeta$ Oph.
\end{abstract}

Subject headings: astrochemistry — ISM: abundances — ISM: molecules — stars: individual ( $\rho$ Ophiuchi A, $\chi$ Ophiuchi)

\section{INTRODUCTION}

Carbon monoxide is the second most abundant molecule, after $\mathrm{H}_{2}$, in interstellar clouds and is seen in dark cloud envelopes through observations at ultraviolet wavelengths (Smith \& Stecher 1971; Morton 1975). High-quality spectra acquired with the Goddard High Resolution Spectrograph (GHRS) on the Hubble Space Telescope allow detailed studies of the $\mathrm{CO}$ abundance and the relative abundances of the various forms containing carbon and oxygen isotopes (Sheffer et al. 1992; Lambert et al. 1994; Lyu, Smith, \& Bruhweiler 1994). For instance, Lambert et al. deduced the relative abundances of ${ }^{12} \mathrm{C}^{16} \mathrm{O},{ }^{13} \mathrm{C}^{16} \mathrm{O},{ }^{12} \mathrm{C}^{18} \mathrm{O}$, and ${ }^{12} \mathrm{C}^{17} \mathrm{O}$. One goal of these studies is to extract information on physical conditions for the gas probed by $\mathrm{CO}$ absorption. The earlier efforts with GHRS of Sheffer et al. (1992), Lambert et al. (1994), and Lyu et al. (1994) had as a focus the sight line toward $\zeta$ Oph $\left(\right.$ R.A. $=16^{\mathrm{h}} 37^{\mathrm{m}} 10^{\mathrm{s}}$; decl. $=-10^{\circ} 34^{\prime} 02^{\prime \prime}$ [J2000]); here we present results on two other sight lines, $\rho$ Oph A (R.A. $=16^{\mathrm{h}} 25^{\mathrm{m}} 35^{\mathrm{s}} ;$ decl. $\left.=-23^{\circ} 26^{\prime} 49^{\prime \prime}[\mathrm{J} 2000]\right)$

\footnotetext{
1 Based on observations obtained with the NASA/ESA Hubble Space Telescope through the Space Telescope Science Institute, which is operated by the Association of Universities for Research in Astronomy, Inc., under NASA contract NAS5-26555.

${ }^{2}$ Department of Physics and Astronomy, University of Toledo, Toledo, OH 43606.

${ }^{3}$ Guest Observer, McDonald Observatory, University of Texas at Austin.

${ }^{4}$ Department of Astronomy, University of Texas, Austin, TX 78712.

${ }^{5}$ Department of Astronomy and Astrophysics, Villanova University, Villanova, PA 19085

6 Deceased.

${ }^{7}$ Jet Propulsion Laboratory, California Institute of Technology, Pasadena, CA 91109.

${ }^{8}$ Department of Physics and Astronomy, Johns Hopkins University, Baltimore, MD 21218.

${ }_{9}^{9}$ Sterrewacht Leiden, P.O. Box 9513, 2300 RA Leiden, Netherlands
}

and $\chi$ Oph (R.A. $=16^{\mathrm{h}} 27^{\mathrm{m}} 01^{\mathrm{s}} ;$ decl. $\left.=-18^{\circ} 27^{\prime} 22^{\prime \prime}[\mathrm{J} 2000]\right)$, in the same portion of the sky.

From observations of dark cloud cores via $\mathrm{CO}$ emission (e.g., Penzias 1981; Langer \& Penzias 1993) and of diffuse clouds via $\mathrm{CH}^{+}$absorption (Stahl et al. 1989; Centurion \& Vladilo 1991; Crane, Hegyi, \& Lambert 1991; Stahl \& Wilson 1992; Vladilo, Centurion, \& Cassola 1993; Centurion, Cassola, \& Vladilo 1995), ambient isotopic ratios for $\mathrm{C}$ and $\mathrm{O}$ can be obtained because $\mathrm{CO}$ in cloud cores and $\mathrm{CH}^{+}$in diffuse clouds are not fractionated. (Throughout this paper we consider [chemical] fractionation to be any process that alters isotopic ratios from ambient values.) In cloud cores the severe attenuation of ultraviolet radiation removes all possible routes for fractionation, while the nonthermal conditions leading to $\mathrm{CH}^{+}$ production are believed to equilibrate $\mathrm{CH}^{+}$isotopomers. The ratios found from these studies of material in the solar neighborhood are ${ }^{12} \mathrm{C} /{ }^{13} \mathrm{C} \sim 65,{ }^{16} \mathrm{O} /{ }^{18} \mathrm{O} \sim 500$, and ${ }^{16} \mathrm{O} /{ }^{17} \mathrm{O} \sim 2600$. These ${ }^{12} \mathrm{C} /{ }^{13} \mathrm{C}$ and ${ }^{16} \mathrm{O} /{ }^{18} \mathrm{O}$ ratios are consistent with the recommendations of Wilson \& Rood (1994), who claimed that $\mathrm{H}_{2} \mathrm{CO}$ gives a lower limit and $\mathrm{CO}$ an upper limit. Wilson \& Rood obtained respective average values of $77 \pm 7$ and $560 \pm 25$. The measurements reported here also probe material near the Sun.

In cloud envelopes, however, the isotopic ratios in $\mathrm{CO}$ are altered by chemical fractionation. Isotopic exchange reactions $\left({ }^{13} \mathrm{C}^{+}+{ }^{12} \mathrm{CO} \leftrightarrow{ }^{12} \mathrm{C}^{+}+{ }^{13} \mathrm{CO}\right)$ can enhance the abundance of ${ }^{13} \mathrm{CO}$ relative to ${ }^{12} \mathrm{CO}$ when gas temperatures are low (Watson, Anicich, \& Huntress 1976) because ${ }^{13} \mathrm{CO}$ has a lower zero-point energy. On the other hand, the more abundant variants are enhanced relative to less abundant ones when selective isotopic photodissociation dominates (Bally \& Langer 1982; Chu \& Watson 1983). Since CO photodissociation takes place via line absorption, the more abundant forms of $\mathrm{CO}$ have lines that are more optically thick, resulting in self-shielding against further 
photodissociation for these isotopic variants. Van Dishoeck \& Black (1988), Kopp et al. (1996), and Warin, Benayoun, \& Viala (1996) incorporated these processes into detailed models, and here we compare the predictions of these models with our observations.

In our earlier studies of $\mathrm{CO}$ fractionation in the diffuse clouds toward $\zeta$ Oph (Sheffer et al. 1992; Lambert et al. 1994), significant enhancement of ${ }^{12} \mathrm{C}^{16} \mathrm{O}$ relative to ${ }^{13} \mathrm{C}^{16} \mathrm{O}$ was found, with a ratio about a factor of 2 larger than the interstellar value of 65-70. Lyu et al. (1994), from the same data set analyzed by Sheffer et al., derived a much smaller ${ }^{12} \mathrm{C}^{16} \mathrm{O} /{ }^{13} \mathrm{C}^{16} \mathrm{O}$ ratio that was indistinguishable from the ambient value. One potential cause for the different conclusions comes from the set of oscillator strengths ( $f$-values) for the $A-X$ system of bands used to extract column densities. The analysis of Lambert et al. (1994) was based on the measurements of Chan, Cooper, \& Brion (1993) from electron energy loss spectra, while the work of Lyu et al. (1994) adopted the $f$-values of Eidelsberg et al. (1992) from absorption measurements with a synchrotron source. Lambert et al. (1994) noted that their data with a higher signal-tonoise ratio yielded a more satisfactory curve of growth when the results of Chan et al. (1993) were utilized. Recent laboratory results (Smith et al. 1994; Federman et al. 1997b; Jolly et al. 1997; Zhong et al. 1997; Stark et al. 1998; Eidelsberg et al. 1999), based on a variety of experimental techniques including absorption of synchrotron radiation, are consistent with those of Chan et al., and therefore the appropriate set of $f$-values to use is no longer an issue. Furthermore, Lambert et al. derived ${ }^{12} \mathrm{C}^{16} \mathrm{O} /{ }^{12} \mathrm{C}^{18} \mathrm{O}$ and ${ }^{12} \mathrm{C}^{16} \mathrm{O} /{ }^{12} \mathrm{C}^{17} \mathrm{O}$ ratios that were also about a factor of 2 greater than the ambient ratios for the oxygen isotopes, a result expected if their conclusions about carbon fractionation were correct. Still, it is unsettling that two groups reached such different conclusions about $\mathrm{CO}$ fractionation, and one of the reasons for acquiring the observations toward $\rho$ Oph A and $\chi$ Oph was to compare the predictions of van Dishoeck \& Black (1988) for diffuse clouds with differing physical conditions. As described below, this objective was met.

Ratios of the $\mathrm{CO}$ isotopic variants in diffuse and translucent clouds have been obtained via millimeter-wave techniques as well. Langer, Glassgold, \& Wilson (1987) mapped a region around $\zeta \mathrm{Oph}$ in ${ }^{12} \mathrm{CO}$ and presented a tentative detection of ${ }^{13} \mathrm{CO}$ toward the star. Their ${ }^{12} \mathrm{CO} /{ }^{13} \mathrm{CO}$ ratio of about $80_{-10}^{+70}$ is formally consistent with our earlier ultraviolet measure. More recently, Kopp et al. (1996) mapped the gas in the vicinity of $\zeta \mathrm{Oph}$ in both isotopomers and obtained lower limits of about 45-65 for the more diffuse directions. Gredel, van Dishoeck, \& Black (1994) surveyed millimeter-wave emission from southern translucent clouds with $A_{V}$ between 1 and 4 mag. While the ${ }^{12} \mathrm{CO} /{ }^{13} \mathrm{CO}$ ratios of antenna temperature, which may be affected by optical depth in the ${ }^{12} \mathrm{CO}$ line, are low, the ${ }^{12} \mathrm{C}^{16} \mathrm{O} /{ }^{12} \mathrm{C}^{18} \mathrm{O}$ and ${ }^{13} \mathrm{C}^{16} \mathrm{O} /{ }^{12} \mathrm{C}^{18} \mathrm{O}$ ratios are similar to or greater than the ambient interstellar values. The ratios involving the oxygen isotopes suggest that selective isotopic photodissociation is operating in these southern clouds.

It is not a simple matter to compare results from ultraviolet absorption and millimeter-wave emission lines. Absorption samples an infinitesimal pencil beam, while emission is an average over the finite telescope beam. Furthermore, emission lines are more prone to uncertainties in excitation, radiative transfer, and abundance. Liszt \& Lucas (1998) overcame these difficulties by measuring millimetric absorption against background compact extragalactic sources. They found a ${ }^{12} \mathrm{CO} /{ }^{13} \mathrm{CO}$ ratio between 15 and 54 that decreases with increasing $N\left({ }^{12} \mathrm{CO}\right)$. Such a result indicates that isotope exchange prevails in their sample of relatively diffuse clouds with $N\left({ }^{12} \mathrm{CO}\right)$ as large as $2 \times 10^{16}$ $\mathrm{cm}^{-2}$.

The paper is organized in the following manner. The next section provides details of the ultraviolet measurements acquired with GHRS as well as ground-based observations of $\mathrm{CH}^{+}$, which provided the ambient ${ }^{12} \mathrm{C} /{ }^{13} \mathrm{C}$ ratio. Section 3 presents our results, while $\S 4$ describes our analysis. In the latter section, results on the excitation of the fine-structure levels in the ground state of $\mathrm{C} \mathrm{I}$ and on absorption from vibrationally excited $\mathrm{H}_{2}$ are included as an aid in constraining the appropriate physical conditions for the clouds. The data on $\mathrm{C}$ I and $\mathrm{H}_{2}$ were extracted from the spectra used for $\mathrm{CO}$ absorption. The resulting column densities for $\mathrm{C} \mathrm{I}$ appear in Zsargó, Federman, \& Cardelli (1997), who derived a self-consistent set of $\mathrm{C}$ I $f$-values for future analyses. (The recent update by Federman \& Zsargó 2001 does not alter these column densities because column densities and Doppler parameters were inferred from lines with well-known $f$-values.) The relative populations in the finestructure levels are used to derive estimates for gas density and temperature (e.g., Jenkins, Jura, \& Loewenstein 1983; Lambert et al. 1994) in the present work. Estimates of the flux of ultraviolet radiation permeating the gas are possible from analyses of vibrationally excited $\mathrm{H}_{2}$ (Federman et al. 1995; Meyer et al. 2001). Next, simple arguments highlight the importance of selective isotopic photodissociation for the three Sco-Oph sight lines. General comparisons with the theoretical predictions of van Dishoeck \& Black (1988), Kopp et al. (1996), and Warin et al. (1996) are made in $\S 5$, where we suggest areas for further improvement in the theoretical models. Such improvements in our models (van Dishoeck \& Black 1988) are beyond the scope of this paper. Throughout this paper we note specific isotopes as needed; otherwise, the general chemical formula is used. Similarly, explicit notation is given for all $\mathrm{CO}$ bands that are not part of the $A-X$ fourth positive system, which is designated only by $v^{\prime}-0$. An Appendix provides observational results for lines of elements heavier than zinc that are seen in our spectra.

\section{OBSERVATIONS AND DATA REDUCTION}

\subsection{Ultraviolet Measurements}

Only a brief description of our reduction procedure is given here; details can be found in our earlier papers (e.g., Lambert, Sheffer, \& Federman 1995). Spectra were acquired with grating G160M of the GHRS and covered three wavelength intervals: $1252-1294,1385-1426$, and 1443-1488 A. Each exposure consisted of four FP-SPLITs, and several exposures were obtained at each nominal wavelength setting but slightly offset from each other. The most important step in the reduction involved the realization of the highest possible signal-to-noise ratio $(\mathrm{S} / \mathrm{N})$ from the data. We applied the procedures outlined by Cardelli \& Ebbets (1994) for the minimization of noise due to granularity, etc., on each FPSPLIT subexposure. The final spectrum for a wavelength interval was derived from merging the individual FPSPLITs in wavelength space. Table 1 provides a listing of the exposures for each star, as well as other pertinent information, such as central wavelength and the number of 
TABLE 1

GHRS OBSERVATIONS

\begin{tabular}{|c|c|c|c|c|c|c|}
\hline Star & File Name & $\begin{array}{l}\text { Wavelength } \\
\text { (§) }\end{array}$ & Counts & Total Counts & S/N Ratio & CO Bands \\
\hline \multirow[t]{10}{*}{$\rho \mathrm{Oph} \mathrm{A}^{\mathrm{a}}}$. & z2b95106 & 1465.60 & 10547 & $\ldots$ & $\ldots$ & $\ldots$ \\
\hline & z2b95107 & 1467.04 & 10363 & $\ldots$ & $\ldots$ & $\ldots$ \\
\hline & z2b95108 & 1461.30 & 10045 & . & $\ldots$ & $\ldots$ \\
\hline & z2b9510a & 1471.35 & 6445 & 37400 & 193 & $2-0,3-0$ \\
\hline & z2b9510b & 1403.67 & 6983 & $\ldots$ & $\ldots$ & $\ldots$ \\
\hline & z2b9510c & 1405.12 & 6489 & $\ldots$ & $\ldots$ & $\ldots$ \\
\hline & $\mathrm{z} 2 \mathrm{~b} 9510 \mathrm{e}$ & 1408.00 & 5989 & 19461 & 140 & $4-0,5-0$ \\
\hline & z2b9510f & 1271.36 & 8917 & $\ldots$ & $\ldots$ & $\ldots$ \\
\hline & z2b9510g & 1272.83 & 8030 & $\ldots$ & $\ldots$ & $\ldots$ \\
\hline & z2b9510i & 1275.75 & 7786 & 24733 & 157 & $10-0,11-0$ \\
\hline \multirow{10}{*}{$\chi \mathrm{Oph}^{\mathrm{b}}$} & z2b90206 & 1465.60 & 15141 & $\ldots$ & $\ldots$ & $\ldots$ \\
\hline & z2b90207 & 1467.04 & 15388 & $\ldots$ & $\ldots$ & $\ldots$ \\
\hline & z2b90208 & 1461.30 & 15358 & $\ldots$ & $\ldots$ & $\ldots$ \\
\hline & z2b9020a & 1471.35 & 14415 & 60302 & 246 & $2-0,3-0$ \\
\hline & z2b9020b & 1403.67 & 15559 & $\ldots$ & $\ldots$ & $\ldots$ \\
\hline & z2b9020c & 1405.12 & 14975 & $\ldots$ & $\ldots$ & $\ldots$ \\
\hline & z2b9020e & 1408.00 & 15279 & 45813 & 214 & $4-0,5-0$ \\
\hline & z2b9020f & 1271.36 & 22992 & $\ldots$ & $\ldots$ & $\ldots$ \\
\hline & z2b9020g & 1272.83 & 17850 & $\ldots$ & $\ldots$ & $\ldots$ \\
\hline & z2b9020h & 1275.75 & 23780 & 64622 & 254 & $10-0,11-0$ \\
\hline
\end{tabular}

a GHRS exposures of HD 147933 with G160M conducted on 1995 June 17.

b GHRS exposures of HD 148184 with G160M conducted on 1995 February 11-12.

counts. The total number of counts for an interval, the $\mathrm{S} / \mathrm{N}$ attained in the merged spectra, and the $\mathrm{CO}$ bands covered by the interval are also shown. The exposure times were $1632 \mathrm{~s}$, except for z2b9020g, where one FP-SPLIT subexposure failed. The resultant $\mathrm{S} / \mathrm{N}$ in our final spectra is on average very similar to the tabulated values computed from (total counts) $^{0.5}$.

Two angstrom segments centered on each $\mathrm{CO}$ band were extracted, and the stellar continuum was rectified with routines available in the IRAF environment distributed by NOAO. The rectification process still retained residuals that constitute the largest errors in the profiles of the strong bands $\left(v^{\prime}=2-5\right)$. While uncertainties in equivalent width $\left(W_{\lambda}\right)$ as large as $25 \%$ are possible, the self-consistent column densities for the suite of bands suggest smaller systematic errors. A further problem arose while we were rectifying the spectra of $\chi \mathrm{Oph}$. Since $\chi \mathrm{Oph}$ is a Be star, its continuum varies much more than do those of $\rho$ Oph A and $\zeta$ Oph. This introduced the largest source of error in the band profiles for the star because the acceptable rectified continuum depends to some extent on the person performing the task. Our final continua reflect the consensus of at least two rectifiers (Y. S. and S. R. F.). Figures 1 and 2 show respective spectra revealing absorption from ${ }^{12} \mathrm{CO}$ and ${ }^{13} \mathrm{CO}$ toward our targets. The observations are indicated by filled circles, and the fits (described below) to extract the ${ }^{12} \mathrm{CO} /{ }^{13} \mathrm{CO}$ ratio are shown as solid lines. Table 2 displays the $W_{\lambda}$ values for the $\mathrm{CO}$ bands derived from the fully reduced spectra of $\rho$ Oph A and $\chi \mathrm{Oph}$. These include the intersystem bands, $a^{\prime}-X(14-0)$ and $e-X(5-0)$. The rms variation in the continuum and the number of pixels across a band were employed in a conservative computation of the uncertainty in $W_{\lambda}$. The CO bands in the spectra of $\rho$ Oph A are much stronger (optically thicker) than those of $\chi$ Oph and $\zeta$ Oph.
Absorption from $\mathrm{C}$ I and vibrationally excited $\mathrm{H}_{2}$ were seen in our spectra. The derivation of $W_{\lambda}$ for lines of neutral carbon toward both stars is presented in Zsargó et al. (1997). For absorption from $v^{\prime \prime}=3$ in the lowest electronic state of $\mathrm{H}_{2}$, we employed a strategy much like the one discussed above for $\mathrm{CO}$. Absorption involving the $R(0), R(1)$, $R(2), R(3), P(2)$, and $P(3)$ lines was clearly detected in the spectrum of $\rho$ Oph A (see Fig. 3). The presence of the $P(2)$ line manifested itself by an asymmetry in the line at $1279.478 \AA$ of $\mathrm{C}$ I from $J=2$. Vibrationally excited $\mathrm{H}_{2}$ was not detected toward $\chi$ Oph. The results for measures of $W_{\lambda}$ appear in Table 3, which includes the results of Federman et al. (1995) for $\zeta$ Oph for reference. Our observations of all the expected lines in essence provide confirmation of vibrationally excited $\mathrm{H}_{2}$ in diffuse clouds, as suggested earlier by Federman et al. (1995). (The data of Meyer et al.

TABLE 2

CO Measurements

\begin{tabular}{|c|c|c|c|}
\hline \multirow[b]{2}{*}{ Molecule } & \multirow[b]{2}{*}{ CO BAND } & \multicolumn{2}{|c|}{$\begin{array}{c}W_{\lambda} \\
(\mathrm{m} \stackrel{\leftrightarrow}{\mathrm{A}})\end{array}$} \\
\hline & & $\rho \mathrm{Oph} \mathrm{A}$ & $\chi \mathrm{Oph}$ \\
\hline \multirow[t]{8}{*}{${ }^{12} \mathrm{CO}$} & $A-X(2-0)$ & $87.7 \pm 1.6$ & $48.9 \pm 1.2$ \\
\hline & $A-X(3-0)$ & $80.9 \pm 2.0$ & $44.6 \pm 1.2$ \\
\hline & $A-X(4-0)$ & $62.5 \pm 2.0$ & $39.9 \pm 1.2$ \\
\hline & $A-X(5-0)$ & $53.3 \pm 1.9$ & $28.3 \pm 1.6$ \\
\hline & $A-X(10-0)$ & $10.0 \pm 1.4$ & $2.0 \pm 0.6$ \\
\hline & $A-X(11-0)$ & $4.6 \pm 1.6$ & $1.6 \pm 1.0$ \\
\hline & $a^{\prime}-X(14-0)$ & $13.8 \pm 1.5$ & $3.6 \pm 0.5$ \\
\hline & $e-X(5-0)$ & $3.6 \pm 0.9$ & $0.8 \pm 0.6$ \\
\hline \multirow[t]{4}{*}{${ }^{13} \mathrm{CO}$} & $A-X(2-0)$ & $10.3 \pm 1.5$ & $2.6 \pm 1.0$ \\
\hline & $A-X(3-0)$ & $11.0 \pm 1.6$ & $2.2 \pm 1.1$ \\
\hline & $A-X(4-0)$ & $4.9 \pm 1.3$ & $1.5 \pm 0.8$ \\
\hline & $A-X(5-0)$ & $4.1 \pm 1.0$ & $0.8 \pm 1.0$ \\
\hline
\end{tabular}



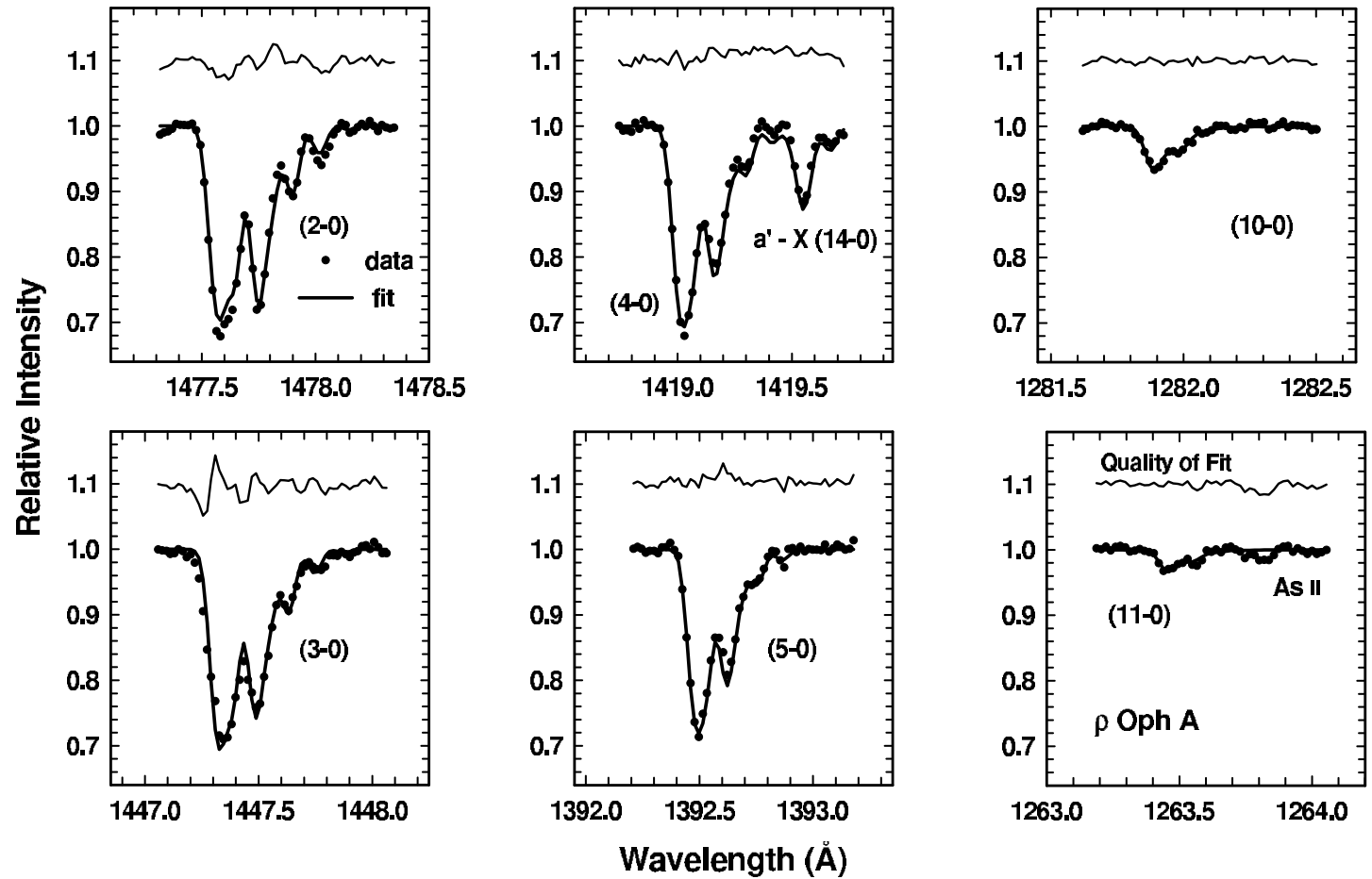

FIG. $1 a$
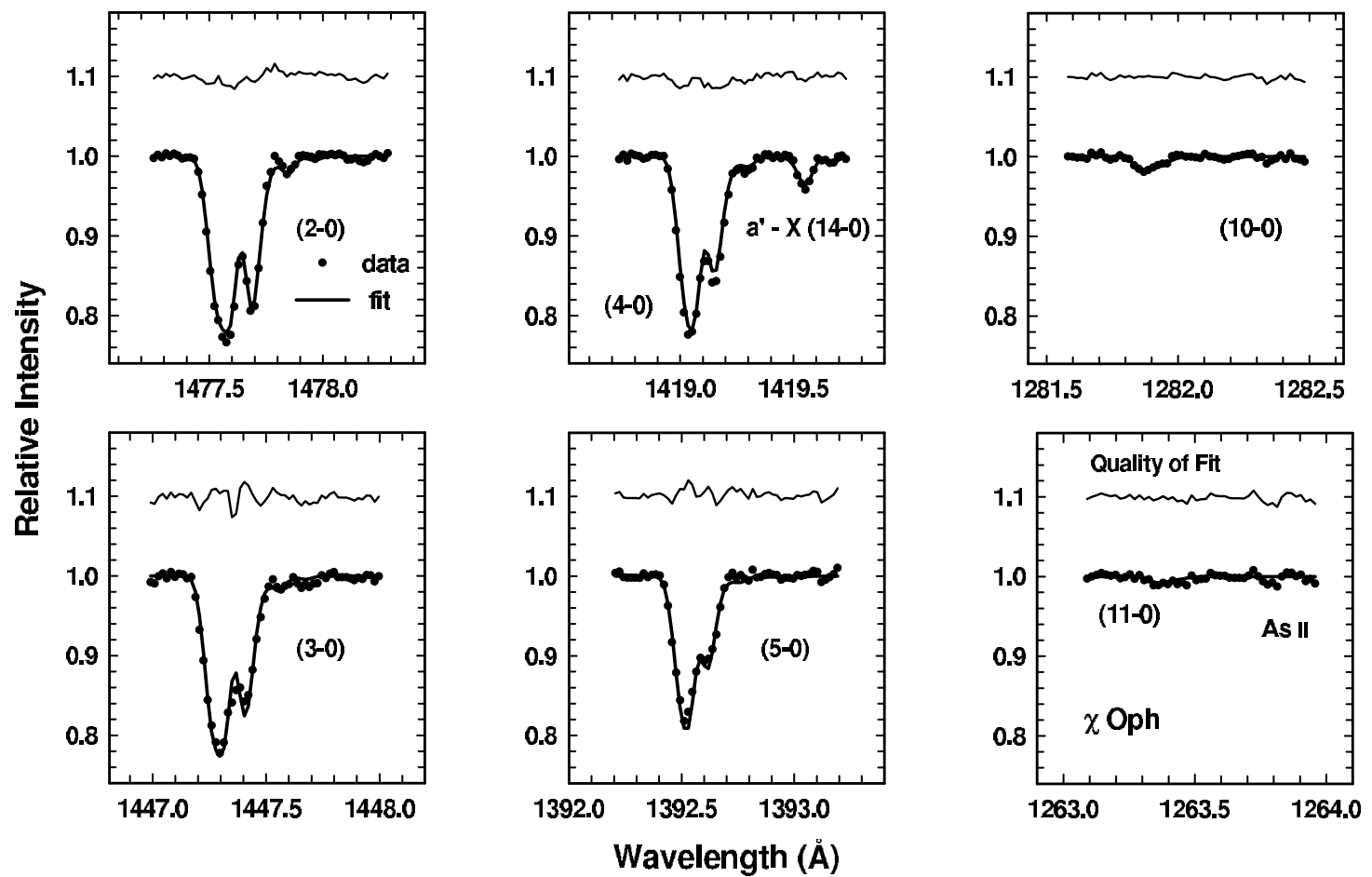

FIG. $1 b$

FIG. 1.-(a) Spectra of ${ }^{12} \mathrm{CO}$ absorption toward $\rho$ Oph A. The data are represented by the filled circles. Our best fit to the data (solid line) and the data - fit (line, offset to 1.10) are also shown. The fit to the $a^{\prime}-X(14-0)$ intersystem band was not used in the derivation of the parameters given in Table 5. Absorption from As II is seen in the spectrum for the $11-0$ band. (b) Spectra for $\chi$ Oph.

2001 are likely probing gas under more extreme conditions.) A steeply varying stellar continuum prevented the derivation of upper limits for $R(0)$ and $R(1)$ toward $\chi \mathrm{Oph}$; furthermore, while the lack of an asymmetry in the $\mathrm{C}$ I line at $1279.478 \AA$ indicated that $P(2)$ is not present, a useful upper limit could not be obtained.

\subsection{Ground-based Measurements}

High-resolution observations of ${ }^{12} \mathrm{CH}^{+}$and ${ }^{13} \mathrm{CH}^{+}$ absorption toward $\rho$ Oph A and $\chi$ Oph were obtained at McDonald Observatory with an echelle spectrograph on the 2.7 m Harlan J. Smith Telescope in 1995 May. The stellar 


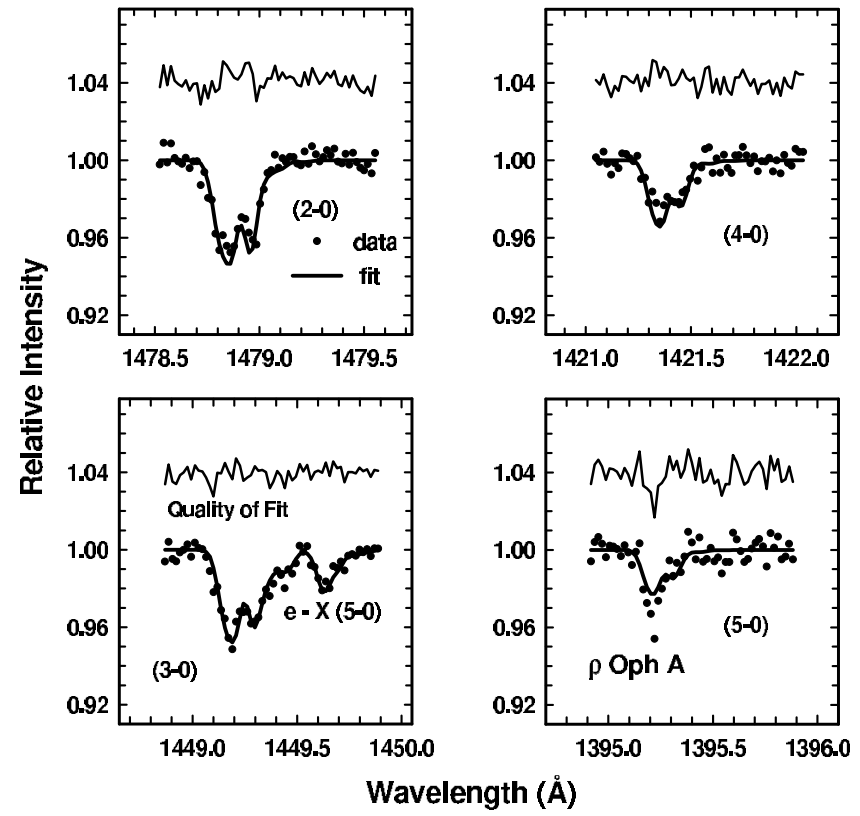

FIG. $2 a$

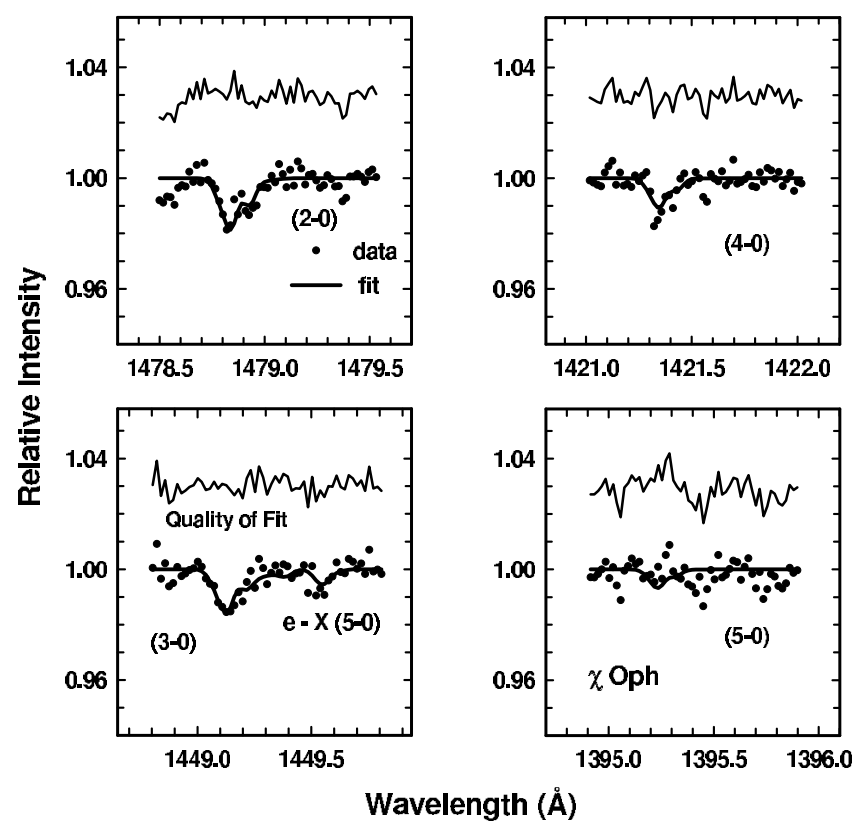

FIG. $2 b$

FIG. 2.-(a) Spectra of ${ }^{13} \mathrm{CO}$ absorption toward $\rho$ Oph A. The data are represented by the filled circles. Our best fit to the data (solid line) and the data - fit (line, offset to 1.04) are also shown. The fit to the $e-X(5-0)$ intersystem band was not used in the derivation of the parameters given in Table 5. (b) Spectra for $\chi \mathrm{Oph}$, except here the quality of the fit is offset to 1.03 .

spectra were imaged onto a Texas Instruments CCD with $15 \mu \mathrm{m}$ pixels. An interference filter was used to limit the spectral coverage to the single order containing the lines near $4230 \AA$. Bias and flat-field images were acquired each night, and Th-Ar comparison spectra were interspersed among the stellar spectra. Dark frames were taken the first night as a check on background levels during the longest exposure. In all, $\rho$ Oph A $(B=5.26)$ was observed for $20 \mathrm{hr}$ and $\chi \operatorname{Oph}(B=4.70)$ for $14 \mathrm{hr}$.

The raw data were reduced with standard IRAF procedures. After bias subtraction, flat-fielding, and removal of

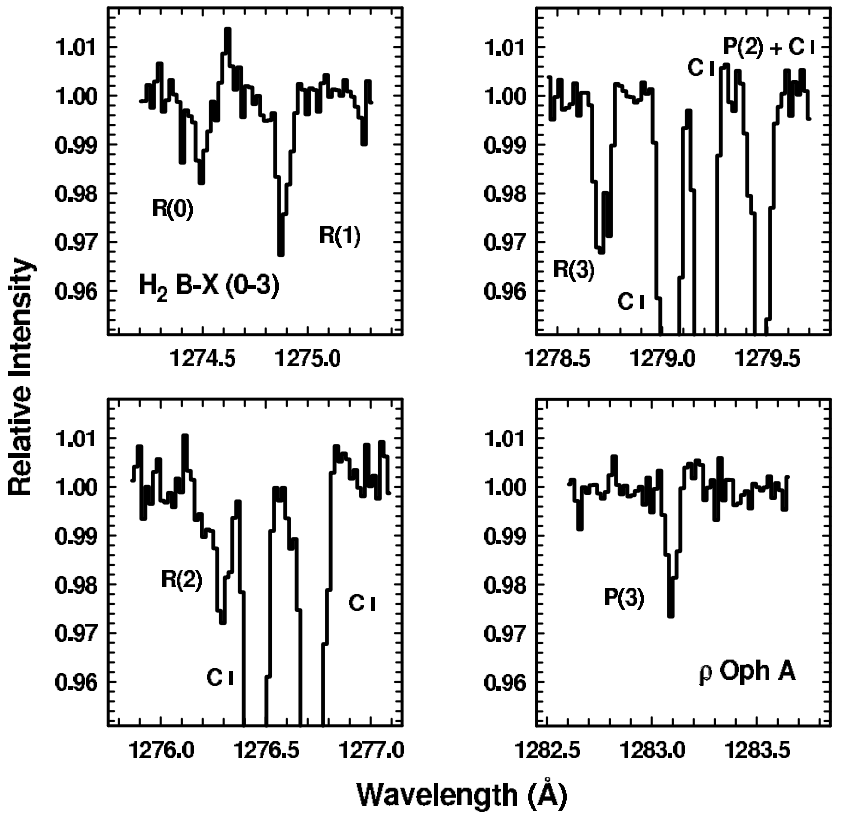

FIG. 3.- Spectra showing absorption from vibrationally excited $\mathrm{H}_{2}$ toward $\rho$ Oph A. The strong lines are due to $\mathrm{C}$ I absorption.

scattered light and cosmic rays, the spectra were extracted. Wavelength calibration was based on lines seen in the ThAr comparison spectra. The spectral resolution, which was derived from the FWHM of the Th-Ar lines averaged over all the nights, was determined to be $33.1 \pm 0.3 \mathrm{m \AA}$, which is significantly larger than the thermal width for the Th lines $(\approx 2 \mathrm{~m} \AA$ ). For $\rho$ Oph A, fitting of the continuum level around the $\mathrm{CH}^{+}$lines was straightforward. However, for $\chi$ Oph, an underlying stellar emission line caused some concern. Centurion et al. (1995, hereafter CCV95) used a synthetic stellar line (which they assign to Fe II $\lambda$ 4233.167) to extract and normalize the spectrum of the star HD 110432 in the direction of the Southern Coalsack. An unfortunate fact of our spectrum was that only part of the stellar line was included, so we performed a normal continuum division, restricted to a limited wavelength interval in order to minimize the influence of baseline structure. Since for $\chi$ Oph the interstellar line is located on the blue wing of the stellar line, rather than in the self-reversal core (cf. CCV95),

TABLE 3

Results For VibRationally ExCited $\mathrm{H}_{2}$

\begin{tabular}{|c|c|c|c|}
\hline \multirow[b]{2}{*}{ LINE } & \multicolumn{3}{|c|}{$\begin{array}{c}W_{\lambda} \\
(\mathrm{m \AA})\end{array}$} \\
\hline & $\rho \mathrm{Oph} \mathrm{A}^{\mathrm{a}}$ & $\chi \mathrm{Oph}$ & $\zeta \mathrm{Oph}^{\mathrm{b}}$ \\
\hline$R(0) \ldots \ldots \ldots \ldots \ldots$ & $1.07 \pm 0.19$ & . & $0.34 \pm 0.16$ \\
\hline$R(1) \ldots \ldots \ldots \ldots \ldots$ & $2.09 \pm 0.27$ & . & $0.33 \pm 0.16$ \\
\hline$R(2) \ldots \ldots \ldots \ldots \ldots$ & $2.00 \pm 0.35$ & $\leq 0.52$ & $\leq 0.30$ \\
\hline$R(3) \ldots \ldots \ldots \ldots \ldots$ & $2.12 \pm 0.35$ & $\leq 0.68$ & $\ldots$ \\
\hline$P(1) \ldots \ldots \ldots \ldots \ldots$ & $\leq 0.70$ & ... & $\ldots$ \\
\hline$P(2) \ldots \ldots \ldots \ldots \ldots$ & $0.81 \pm 0.26$ & $\ldots$ & $\cdots$ \\
\hline$P(3) \ldots \ldots \ldots \ldots \ldots$ & $1.43 \pm 0.28$ & $\leq 0.53$ & $\ldots$ \\
\hline
\end{tabular}

a Weighted average of independent determinations by J. A. C. and S. R. F.

b From Federman et al. 1995. 


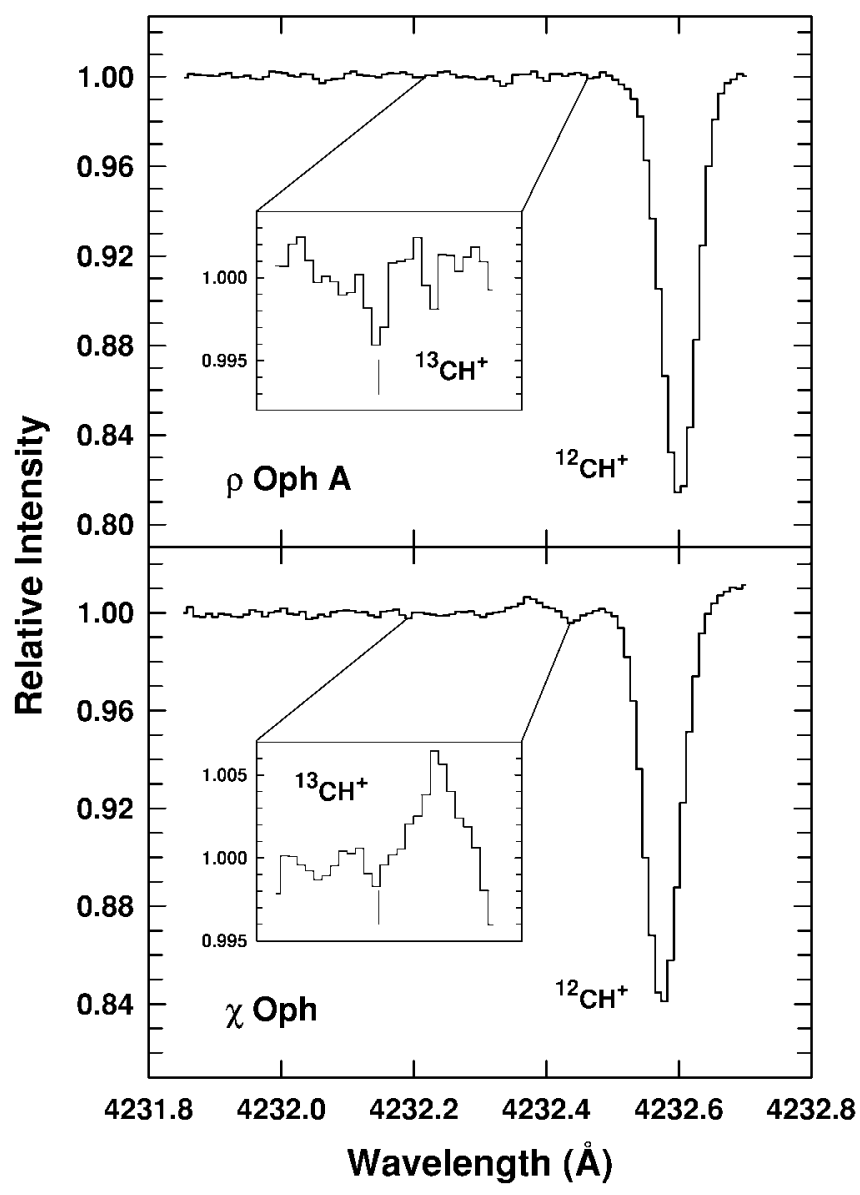

FIG. 4.- Rectified spectra of $\mathrm{CH}^{+}$absorption toward $\rho$ Oph A and $\chi$ Oph. The inserts focus on the region of ${ }^{13} \mathrm{CH}^{+}$absorption.

we do not believe that our extraction procedure added significantly to the derived uncertainties. The rms dispersion in the rectified continuum yielded an $\mathrm{S} / \mathrm{N}$ per pixel of about 650 for both stars. The rectified spectra appear in Figure 4.

After normalization/rectification, the $W_{\lambda}$ for the ${ }^{12} \mathrm{CH}^{+}$ and ${ }^{13} \mathrm{CH}^{+}$lines were derived from Gaussian fits. Two methods utilizing the STSDAS routine NGAUSS were employed. In the first, we adopted an isotope shift of $-0.272 \AA$, as measured from the ${ }^{12} \mathrm{CH}^{+}$line. This value is based on the laboratory measurements by Bembenek (1997) for ${ }^{13} \mathrm{CH}^{+}$and those of Carrington \& Ramsay (1982) for ${ }^{12} \mathrm{CH}^{+}$. We kept the widths of the ${ }^{13} \mathrm{CH}^{+}$and ${ }^{12} \mathrm{CH}^{+}$lines the same in our syntheses. The results of this analysis appear in Table 4 , where $W_{\lambda}, v_{\mathrm{LSR}}$, and the Doppler parameter ( $b$-value) are given. The LSR velocities obtained here are

TABLE 4

Results FOR ${ }^{12} \mathrm{CH}^{+}$AND ${ }^{13} \mathrm{CH}^{+}$

\begin{tabular}{ccrcc}
\hline \hline Star & Molecule & \multicolumn{1}{c}{$\begin{array}{c}W_{\lambda} \\
(\mathrm{m \AA})\end{array}$} & $\begin{array}{c}v_{\text {LSR }} \\
\left(\mathrm{km} \mathrm{s}^{-1}\right)\end{array}$ & $\begin{array}{c}b \\
\left(\mathrm{~km} \mathrm{~s}^{-1}\right)\end{array}$ \\
\hline$\rho$ Oph A .......... & ${ }^{12} \mathrm{CH}^{+}$ & $12.25 \pm 0.09$ & 4.5 & 2.2 \\
& ${ }^{13} \mathrm{CH}^{+}$ & $0.11 \pm 0.05$ & $\ldots$ & 2.2 \\
$\chi$ Oph.............. & ${ }^{12} \mathrm{CH}^{+}$ & $10.45 \pm 0.16$ & 2.6 & 2.1 \\
& ${ }^{13} \mathrm{CH}^{+}$ & $0.17 \pm 0.05$ & $\ldots$ & 2.1 \\
\hline
\end{tabular}

Note.- Results based on separation of $-0.272 \AA$, and the line width for ${ }^{13} \mathrm{CH}^{+}$was constrained to be the same as that for ${ }^{12} \mathrm{CH}^{+}$. within $1 \mathrm{~km} \mathrm{~s}^{-1}$ of those derived by Crane, Lambert, \& Sheffer (1995) from ultra-high-resolution observations; the $b$-values also agree very well. The relatively large $b$-values seen for $\mathrm{CH}^{+}$lines are likely a consequence of $\mathrm{CH}^{+}$production involving an endothermic reaction (e.g., Lambert \& Danks 1986). As for the $W_{\lambda}$, our determinations and those of Lambert \& Danks (1986) are very similar, as are the results of Crane et al. for $\chi \mathrm{Oph}$. For $\rho \mathrm{Oph} \mathrm{A}$, the measurements of Crane et al. yield a $W_{\lambda}$ about $2 \sigma$ larger. The values of $W_{\lambda}$ yield column density ratios, $N\left({ }^{12} \mathrm{CH}^{+}\right) / N\left({ }^{13} \mathrm{CH}^{+}\right)$, of $120 \pm 54$ and $65 \pm 19$, respectively, for the gas toward $\rho$ Oph A and $\chi$ Oph from a curve-of-growth analysis with $f=5.5 \times 10^{-3}$ and $b=2.5 \mathrm{~km} \mathrm{~s}^{-1}$. Use of a smaller $b$-value, $2 \mathrm{~km} \mathrm{~s}^{-1}$, introduces a negligible change because the optical depth at line center for ${ }^{12} \mathrm{CH}^{+}$is less than 0.5 . The uncertainties in the ratios are dominated by the precision of the ${ }^{13} \mathrm{CH}^{+}$measures. The result for $\chi \mathrm{Oph}$ is in the range (60-70) determined for other sight lines, but that for $\rho$ Oph A is a bit high (consistent at the $1 \sigma$ level, however).

In the second method, we fixed the relative wavelength offset between the isotopomers at $-0.265 \AA$. This isotopic shift is based on the measurements of CCV95 for $\mathrm{CH}^{+}$ toward HD 110432, rather than the value of $-0.26 \AA$ obtained from theoretical calculations by Auguson \& Herbig (1967) and used in earlier studies (e.g., Centurion \& Vladilo 1991). The quoted uncertainty of about 2-3 mA in each laboratory measurement (Carrington \& Ramsay 1982; Bembenek 1997) indicates that the shift derived from astronomical spectra is consistent with the laboratory value at about the $1.5 \sigma$ level. With a shift of $-0.265 \AA$ we find $109 \pm 36$ and $61 \pm 20$ for $\rho$ Oph A and $\chi$ Oph. In summary, the ratios derived here indicate that the ${ }^{12} \mathrm{C} /{ }^{13} \mathrm{C}$ ratio is approximately 65 , as in other, more precise determinations for the general interstellar medium (e.g., Langer \& Penzias 1993; CCV95) and for the $\rho$ Oph molecular cloud in particular (Bensch et al. 2001).

\section{RELATIVE ABUNDANCES OF CO ISOTOPOMERS}

Three steps were employed in the extraction of the relative abundances among the isotopic variants of $\mathrm{CO}$. The extraction was based on synthesis of the measured bands through the minimization of the rms difference between the observed band and the fit. The $f$-values of Chan et al. (1993) were adopted, as was the case in Lambert et al. (1994). The first step involved fits to absorption from the weaker 10-0 and $11-0$ bands in order to set the initial column density. For this column density, simultaneous fits of the stronger bands yielded rotational excitation temperatures $\left(T_{\text {rot }}=\right.$ $T_{10}, T_{21}$, and $T_{32}$ ) and the $b$-value. The final step involved fitting the ${ }^{13} \mathrm{CO}$ bands, which are not very sensitive to changes in $T_{\text {rot }}$ and $b$-value because these bands are optically thin. As consistency checks on these derived rotational excitation temperatures, $T_{\text {rot }}$ also was estimated from the $10-0$ and $11-0$ bands of ${ }^{12} \mathrm{CO}$ and the $2-0,3-0$, and $4-0$ bands of ${ }^{13} \mathrm{CO}$ under the assumption that $T_{10}=T_{21}=T_{32}$. These determinations yield similar excitation temperatures for ${ }^{12} \mathrm{CO}$, within the mutual uncertainties, and show that the rotational excitation in ${ }^{12} \mathrm{CO}$ and ${ }^{13} \mathrm{CO}$ is essentially the same. One added complication with the $2-0$ and $3-0$ bands for ${ }^{13} \mathrm{CO}$ was the presence of the $\mathrm{C}^{18} \mathrm{O}$ band. For the synthesis of the ${ }^{13} \mathrm{CO}$ bands toward $\rho \mathrm{Oph} \mathrm{A}$, where all bands were much stronger than those toward $\chi \mathrm{Oph}$, the ${ }^{13} \mathrm{C}^{16} \mathrm{O} /$ ${ }^{12} \mathrm{C}^{18} \mathrm{O}$ ratio was inferred as well. Figures 1 and 2 also show 
TABLE 5

Results FROM FitTing the CO Bands

\begin{tabular}{|c|c|c|}
\hline Parameter & $\rho$ Oph A & $\chi \mathrm{Oph}$ \\
\hline$N\left({ }^{12} \mathrm{CO}\right)\left(\mathrm{cm}^{-2}\right) .$. & $(1.92 \pm 0.25) \times 10^{15}$ & $(3.8 \pm 1.0) \times 10^{14}$ \\
\hline$v_{\text {helio }}\left(\mathrm{km} \mathrm{s}^{-1}\right) \ldots \ldots \ldots \ldots \ldots$ & $-4.4 \pm 1.6$ & $-10.3 \pm 1.5$ \\
\hline$b\left(\mathrm{~km} \mathrm{~s}^{-1}\right) \ldots \ldots$ & $0.60 \pm 0.02$ & $0.66 \pm 0.04$ \\
\hline$T_{10}(\mathrm{~K}) \ldots \ldots \ldots \ldots \ldots \ldots \ldots$ & $2.7 \pm 0.1^{\mathrm{a}}$ & $3.0 \pm 0.3^{\mathrm{b}}$ \\
\hline$T_{21}(\mathrm{~K}) \ldots \ldots \ldots \ldots \ldots \ldots$ & $7.6 \pm 0.5^{\mathrm{a}}$ & $5.2 \pm 0.6^{\mathrm{b}}$ \\
\hline$T_{32}(\mathrm{~K}) \ldots \ldots \ldots \ldots \ldots \ldots$ & $8.4 \pm 0.5^{\mathrm{a}}$ & $7.5 \pm 3.7^{b}$ \\
\hline$N\left({ }^{12} \mathrm{CO}\right) / N\left({ }^{13} \mathrm{CO}\right) \ldots \ldots$ & $125 \pm 23$ & $117 \pm 35$ \\
\hline$N\left(\mathrm{C}^{16} \mathrm{O}\right) / N\left(\mathrm{C}^{18} \mathrm{O}\right) \ldots \ldots$ & $1100 \pm 600$ & $\ldots$ \\
\hline
\end{tabular}

a $T_{10}, T_{21}, T_{32}=10.3 \pm 2.9 \mathrm{~K}$ from the $10-0$ and $11-0$ bands, while the 2-0 to $5-0$ bands of ${ }^{13} \mathrm{CO}$ give $7.7 \pm 2.5 \mathrm{~K}$.

b $T_{10}, T_{21}, T_{32}=4.7 \pm 2.2 \mathrm{~K}$ from the $10-0$ and $11-0$ bands, while the $2-0$ to $4-0$ bands of ${ }^{13} \mathrm{CO}$ give $4.4 \pm 2.9 \mathrm{~K}$.

the synthetic spectra and the residuals between the observed and synthetic profiles. For completeness, the data are displayed with theoretical curves of growth, which are based on our results in Table 5, in Figure 5. All data can be described very well by these curves of growth, which were computed for a given $N(\mathrm{CO})$ with the appropriate wavelengths for the various lines comprising the bands. Allowance was also made for absorption from ${ }^{12} \mathrm{C}^{18} \mathrm{O}$ in the strongest bands.

This procedure provided the necessary information for our study of chemical fractionation in $\mathrm{CO}$; the results appear in Table 5. The values for $N\left({ }^{12} \mathrm{CO}\right)$ are the most precise among available ones for the sight lines to $\rho$ Oph A and $\chi$ Oph. We note that the $\mathrm{CO}(A-X)$ bands toward $\rho$ Oph A are stronger than the ones seen in the spectrum of $\zeta$ Oph (Sheffer et al. 1992; Lambert et al. 1994; Lyu et al. 1994). The ${ }^{12} \mathrm{CO}$ column density is not as large, however, because the $b$-values are greater. Our determination of the column density toward $\rho$ Oph A is about a factor of 3 greater than the value inferred from Copernicus data of the $B-X(0-0)$ band at $1150 \AA$ (Snow \& Jenkins 1980). Most of the difference arises from the fact that they adopted a $b$-value of 1.2 $\mathrm{km} \mathrm{s}^{-1}$ where we deduced $b=0.6 \mathrm{~km} \mathrm{~s}^{-1}$. Because we fitted several bands simultaneously, whose wavelengths fall on different parts of the blaze function for G160M, our $b$ values are less susceptible to the variations in spectral resolution across the blaze noted by Lyu et al. (1994). As for $\chi$ Oph, the Copernicus measurements of Frisch (1980) on the $C-X(0-0)$ band near $1088 \AA$, using a band oscillator strength of 0.123 (Federman et al. 2001), yield a value for $N\left({ }^{12} \mathrm{CO}\right)$ that agrees nicely with ours. We also extracted the Copernicus data on $\rho$ Oph A and $\chi$ Oph for both the $B-X$ and $C-X(0-0)$ bands from the MAST archive at the Space Telescope Science Institute and used routines in IRAF for an improved comparison with the GHRS results. Table 6

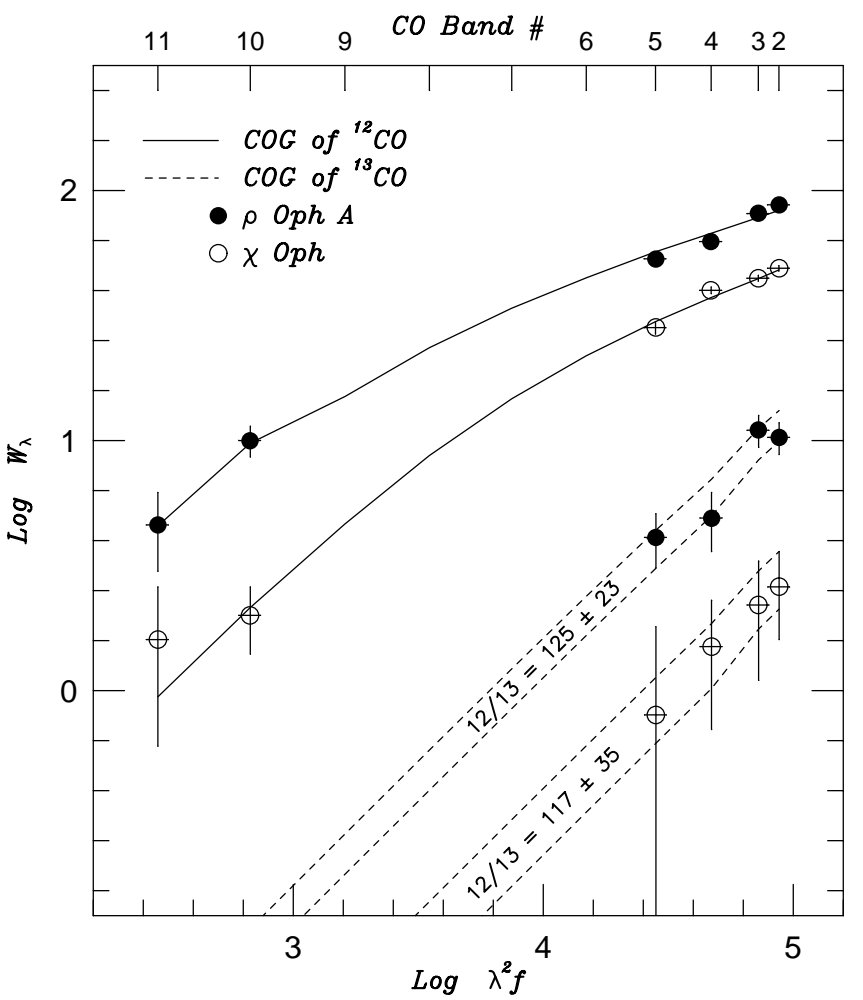

FIG. 5.- Curves of growth for the $A-X$ system of bands in ${ }^{12} \mathrm{CO}$ (solid lines) and ${ }^{13} \mathrm{CO}$ (dashed lines) toward $\rho$ Oph A (filled circles) and $\chi$ Oph (open circles). The numbers along the top give $v^{\prime}$ for the band. The resulting ${ }^{12} \mathrm{CO} /{ }^{13} \mathrm{CO}$ ratios are also indicated.

shows the comparison of measured and derived values of $W_{\lambda}$; the derived values are based on the $f$-values of Federman et al. (2001) and the cloud parameters $\left[N\left({ }^{12} \mathrm{CO}\right)\right.$, $T_{10}, T_{21}$, and $b$-value] from the synthesis of the $A-X$ bands. The correspondence between the values is very good. The relatively slight differences for the $C-X$ band are mainly the result of imprecise subtraction of the $\mathrm{Cl}_{\mathrm{I}}$ line at $1088 \AA$. The $W_{\lambda}$ for the $C-X$ band toward $\rho$ Oph A from Federman et al. (1980) is significantly less than that reported here; an incorrect continuum placement is likely the cause.

While the above comparison shows that the derived cloud parameters are rather robust, we now comment on the velocities, $b$-values, and excitation temperatures in Table 5 . The heliocentric velocities associated with the weighted blend of the $Q(1)$ and $Q(2)$ lines for the 2-0 through 5-0 bands agree with those obtained by Crane et al. (1995) to within about $2 \mathrm{~km} \mathrm{~s}^{-1}$. Since thermal velocities do not contribute significantly to the line width, we would expect similar $b$-values for different species. Indeed, the $b$-values of $\approx 0.60-0.70 \mathrm{~km} \mathrm{~s}^{-1}$ agree with other measures based on

TABLE 6

Comparison of Results For the $B-X$ and $C-X$ Bands

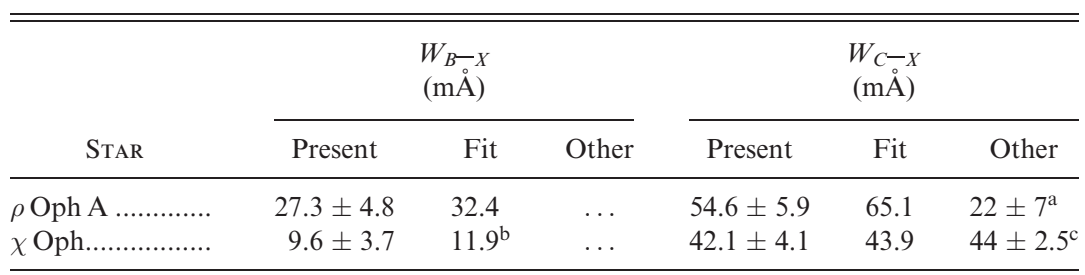

a Federman et al. 1980.

${ }^{\mathrm{b}}$ Since the $P$-branch is within the noise, the $W_{\lambda}$ for the $R$-branch is given.

c Frisch 1980. 
ultra-high-resolution observations. Measurements of K I $\lambda 4044$ toward $\chi$ Oph (Knauth, Federman, \& Lambert 2003) indicate a similar value. The $\mathrm{K}$ I column density from $\lambda 7699$ (Welty \& Hobbs 2001) can be made consistent with the results from $\lambda 4044$ by assuming one velocity component with a $b$-value of about $0.70 \mathrm{~km} \mathrm{~s}^{-1}$. Moreover, Welty \& Hobbs suggest that the strongest component toward $\rho$ Oph A has a $b$-value of approximately $0.60 \mathrm{~km} \mathrm{~s}^{-1}$. For the direction toward $\rho$ Oph A, synthesis of the strong and weak band profiles yielded different rotational excitation temperatures. While the $2-0$ to $5-0{ }^{12} \mathrm{CO}$ bands indicate $T_{10}$ of $2.7 \mathrm{~K}$, the weaker $10-0$ and $11-0$ bands, as well as $2-0$ to $5-0$ bands of ${ }^{13} \mathrm{CO}$, are better fitted with $T_{10}$ between $7.7 \pm 2.5$ and $10.3 \pm 2.9 \mathrm{~K}$. (The larger excitation temperatures are more consistent with $T_{21}$ and $T_{32}$ derived from the strong bands of ${ }^{12} \mathrm{CO}$.) If the effect is substantiated by more precise determinations, it could be understood in terms of how deeply the observations probe into the cloud: optically thin bands, which sample most of the volume, reveal higher excitation temperatures in the core, but saturated bands are sensitive to outside regions of the cloud. This effect can arise from a density gradient, which also appears in our analysis of $\mathrm{C}_{\mathrm{I}}$ levels and vibrationally excited $\mathrm{H}_{2}$ described below. The effect of a decreasing $\mathrm{CO}$ excitation temperature toward the edge of the cloud can also result from less trapping of $\mathrm{CO}$ millimeter lines in the periphery compared with the amount of trapping in the cloud's core (e.g., Bernes 1979; Hogerheijde \& van der Tak 2000). An explicit radiation transfer code would be needed to confirm this scenario. In its stead, we also suggest that some combination of two clouds with differing physical conditions, such as the two dominant components seen by Welty \& Hobbs (2001) in K I absorption, might account for $T_{10}$ variations in the bands. Crane et al. (1995) found two CH components toward $\rho$ Oph A as well, one with a small $b$-value like that inferred by $\mathrm{CO}$ and the other with a larger $b$-value.

Two intersystem bands of $\mathrm{CO}, a^{\prime}-X(14-0)$ and $e-X(5-0)$, are present in our spectra of $\rho \mathrm{Oph} \mathrm{A}$, and the stronger $a^{\prime}-X$ band is seen toward $\chi$ Oph. Table 2 gives the measured values of $W_{\lambda}$. Federman et al. (1994a) derived an $f$-value for the $a^{\prime}-X$ band that was $31 \% \pm 7 \%$ smaller than that computed by Morton \& Noreau (1994), while the $f$-value for the $e-X$ band was $89 \% \pm 16 \%$ smaller. Fitting the bands with the parameters deduced from the $A-X$ bands suggests $f\left(a^{\prime}-X\right)$ of $(0.7 \pm 0.2) f(\mathrm{MN})$ and $f(e-X)$ of $(1.1 \pm 0.3)$ $\times f(\mathrm{MN})$, where $f(\mathrm{MN})$ is the value quoted by Morton \& Noreau (1994). Our more precise determinations from spectra of X Per taken with the Space Telescope Imaging Spectrograph (Sheffer, Federman, \& Lambert 2002) reveal respective $f$-value ratios of $0.94 \pm 0.15$ and $0.78 \pm 0.12$. The combination of the results for these directions suggests that the band oscillator strength quoted by Morton \& Noreau should be decreased some 10\%-30\%. Improved analysis that includes multiple curve crossings (Rostas et al. 2000; Eidelsberg \& Rostas 2003) substantiates the astronomical findings.

\section{ANALYSIS}

\subsection{Density from Atomic and Molecular Excitation}

The relative populations of the fine-structure levels in the ground state of C I yield estimates for gas density and temperature (e.g., Jenkins et al. 1983). The populations are mainly affected by collisional (de)excitation and far-infrared radiative decay and to a lesser extent by ultraviolet pumping and the subsequent decay from the excited electronic level. We previously analyzed the distribution of these levels in our study of the gas toward $\zeta$ Oph (Lambert et al. 1994). Here we apply the same technique to the data on C I toward $\rho$ Oph A and $\chi$ Oph. Zsargó et al. (1997) derived column densities for each level; the resulting ratios, $N\left(J^{\prime}\right) / N(J)$, are $N(1) / N(0)=0.430 \pm 0.024$ and $0.422 \pm 0.019$, respectively, for $\rho$ Oph A and $\chi$ Oph and $N(2) / N(0)=$ $0.238 \pm 0.014$ and $0.235 \pm 0.011$ for the two sight lines. The abundances (relative to total protons) of the collision partners $\left(\mathrm{H}\right.$, ortho- $\mathrm{H}_{2}$, and para- $\left.\mathrm{H}_{2}\right)$ are also needed; these were obtained from Savage et al. (1977). For He, we assumed an abundance of $10 \%$. As in all analyses of excitation, if the temperature is known, the density of collision partners $\left(n_{c}\right)$ is inferred. For neutral interstellar gas, $n_{c} \sim n(\mathrm{H})+n\left(\mathrm{H}_{2}\right)$.

The results of our analysis appear in Figure 6, where the allowed ranges in density and temperature are shown for $2 \sigma$
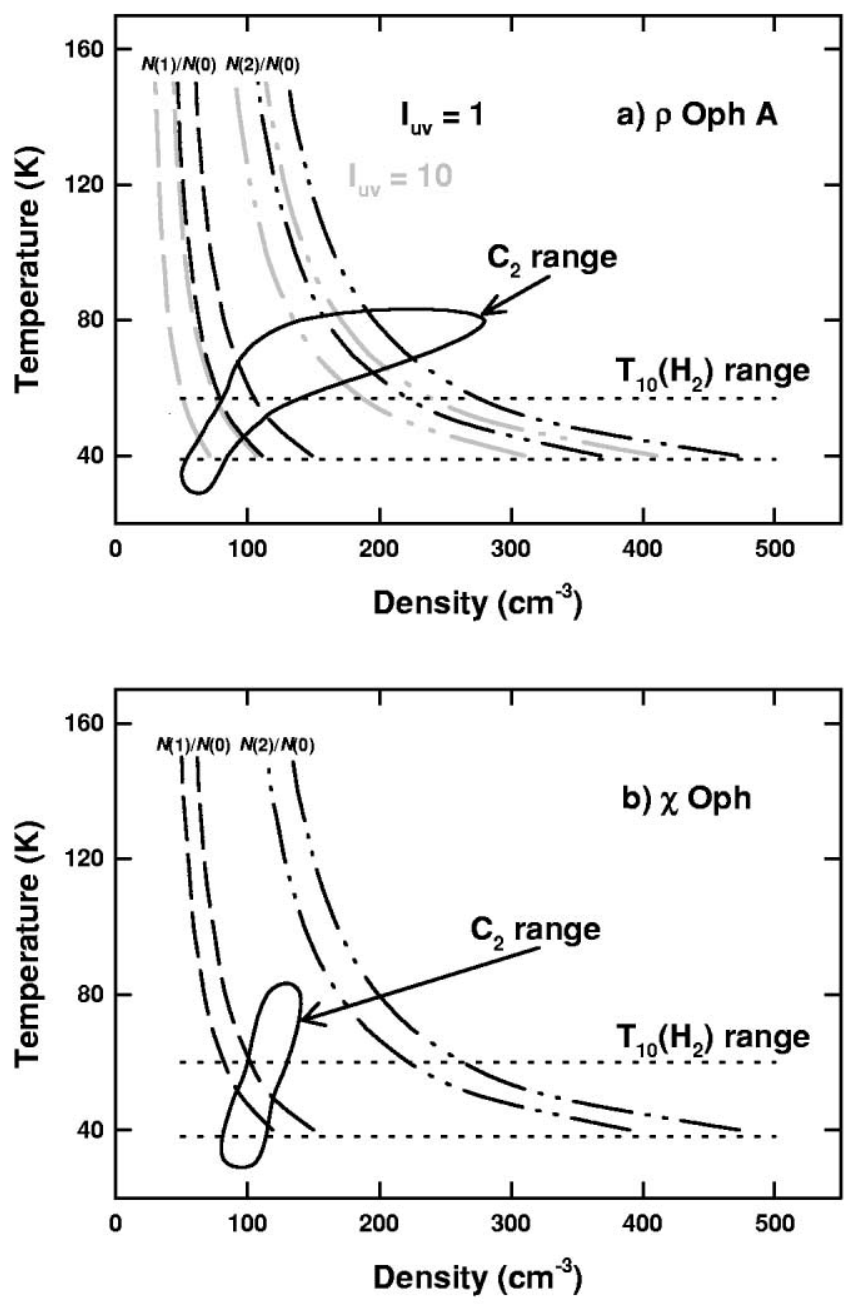

FIG. 6.-Results of excitation analyses for $\mathrm{C}_{\mathrm{I}}$ and $\mathrm{C}_{2}$. The density indicated here refers to collision partners, $n_{c}$. The dashed and dot-dashed curves show the constraints inferred from $N(1) / N(0)$ and $N(2) / N(0)$, respectively, while the solid contour represents the acceptable values from the $\mathrm{C}_{2}$ rotational lines. The range in kinetic temperature deduced from the relative amounts of $\mathrm{H}_{2}$ in $J=0$ and 1 is indicated by dotted lines. For $\rho$ Oph A, the gray curves show the effect of a larger UV flux on $\mathrm{C}$ I excitation. 
excursions about the observed column density ratios. One facet is immediately clear: the results for $N(1) / N(0)$ (dashed lines) suggest lower densities than do the results for $N(2) / N(0)$ (dot-dashed lines). The earlier analysis by Jenkins et al. (1983), based on spectra from the Copernicus satellite, did not reveal this dichotomy. Their column density ratios, although generally consistent with our estimates, probably had uncertainties too large to discern any difference. We also note that Jenkins et al. and Federman, Welty, \& Cardelli (1997c) found this dichotomy toward the nearby line of sight to $\beta^{1} \mathrm{Sco}$, while Zsargó \& Federman (2003) detected the two velocity components in high-resolution ECH-A data on $1 \mathrm{Sco}, \delta$ Sco, and $\sigma$ Sco. Ultra-high-resolution spectra reveal additional, weaker components in $\mathrm{K}$ I absorption toward $\rho$ Oph and $\chi$ Oph (Welty \& Hobbs 2001). Moreover, for our two directions, the $b$-values deduced from curves of growth for the lines originating from $J=0$ and 1 are larger than the $b$-value for the $J=2$ lines (Zsargó et al. 1997). From this information, we infer that absorption from the lower fine-structure levels is probing (1) more extended portions of the neutral gas where the average density is expected to be lower or (2) an additional low-density component.

The use of the rotational excitation temperature for the $J=0$ and 1 levels in $\mathrm{H}_{2}\left[T_{01}\left(\mathrm{H}_{2}\right)\right]$ as a measure of the kinetic temperature allows us to derive values for the gas density. Adopting the $2 \sigma$ range in values of Savage et al. (1977), which are also indicated in Figure 6, yields densities for the gas toward both of our targets of $\approx 100$ and $200-400 \mathrm{~cm}^{-3}$ from $N(1) / N(0)$ and $N(2) / N(0)$, respectively. The estimates are rather robust. There is little change when we apply substantial differences in the relative fractions of collision partners; the inferred densities are mainly influenced by the observed relative fine-structure populations. For $\rho$ Oph A, the effects of increasing the amount of UV pumping are illustrated in Figure 6 as well. A 10-fold increase in UV flux, as suggested by our $\mathrm{H}_{2}$ measurements, lowers the density estimate a small but noticeable amount.

We can derive other estimates for gas density from analysis of the distribution of rotational levels in the ground state of $\mathrm{C}_{2}$ or $\mathrm{CO}$. Excitation of $\mathrm{C}_{2}$ involves a combination of collisions, radiative decay between rotational levels, and near-infrared pumping to the $A$ electronic state, followed by radiative cascades among vibrational and rotational levels of the ground state. According to van Dishoeck \& Black (1982), the amount of excitation can be represented by $n_{c} \sigma / I_{\text {ir }}$, where $\sigma$ is the collisional cross section and $I_{\text {ir }}$ is the enhancement in IR flux over the typical interstellar value. The observational data on $\mathrm{C}_{2}$ of Danks \& Lambert (1983) and van Dishoeck \& de Zeeuw (1984), along with the theoretical predictions of van Dishoeck \& Black (1982), lead to the estimates shown in Figure 6 as enclosed areas. These were obtained through a $\chi^{2}$ minimization procedure, weighted by the precision of the observational data on column density for a given level. We adopted a cross section of $2 \times 10^{-16} \mathrm{~cm}^{-2}$, an oscillator strength of $1 \times 10^{-3}$, and $I_{\mathrm{ir}}$ of 1 .

The results from $\mathrm{C}_{2}$ excitation are generally consistent with those from the $N(1) / N(0)$ ratio for $\mathrm{C}$, which is a bit of a surprise. The estimates from $\mathrm{C}_{2}$ are very sensitive to assumptions concerning excitation. Recent determinations (Langhoff et al. 1990; Erman \& Iwamae 1995; Lambert et al. $1995)$ of the oscillator strength for the $A-X(2-0)$ band, $f_{20}$, are $20 \%-40 \%$ larger than the adopted value. A larger $f$-value would increase the density estimate (van Dishoeck \& Black 1982) a corresponding amount. The adopted cross section is in the middle of the range suggested by van Dishoeck \& Black. Quantal calculations (Lavendy et al. 1991; Robbe et al. 1992; Phillips 1994) span the suggested range, with larger cross sections giving lower densities. A reexamination of $\mathrm{C}_{2}$ excitation, utilizing the more recent determinations of $f_{20}$ and collisional cross sections, appears necessary.

Finally, the excitation of CO can be studied. This excitation is controlled by collisions, radiative decay, absorption of the cosmic background radiation, and resonant scattering of CO lines from nearby molecular clouds (Wannier, Penprase, \& Andersson 1997). The latter process appears to be the dominant one, and as a result, only upper limits on density are possible. This conclusion is based on the $J=1 \rightarrow 0$ maps of the $\mathrm{Sco}-\mathrm{Oph}$ region by de Geus, Bronfman, \& Thaddeus (1990). We selected the channel with $v_{\text {LSR }}$ between 3 and $5 \mathrm{~km} \mathrm{~s}^{-1}$ and estimated the filling factors for the gas at the projected positions of $\rho$ Oph A and $\chi$ Oph. The excitation temperature, $T_{10}$, due solely to resonant scattering of emission from the molecular cloud is $7.3 \pm 1.0$ and $3.2 \pm 0.4 \mathrm{~K}$, respectively. These values are comparable to, or greater than, the values inferred from profile synthesis of the UV absorption bands. Therefore, all that can be said about density is that is it less than about $1000 \mathrm{~cm}^{-3}$. The fact that $T_{10}$ is not larger than $T_{21}$ or $T_{32}$, as would be expected for subthermal conditions in relatively diffuse gas, is further proof that resonant scattering of line radiation dominates $\mathrm{CO}$ excitation along the two sight lines.

\subsection{UV Flux from $\mathrm{H}_{2}$ Excitation}

In cold $(T<1000 \mathrm{~K})$ gas, the population of the $v=3$ level of the ground electronic state is governed by the flux of ultraviolet radiation permeating the cloud (Black \& van Dishoeck 1987). Absorption from the $J=0,1$, and 2 levels in the vibrational ground state - the ones with the greatest populations - of ultraviolet photons leaves the $\mathrm{H}_{2}$ molecules in excited electronic states, but rapid decay returns the molecules to various vibrational levels of the ground electronic state (e.g., Black \& Dalgarno 1976). Collisional (de)excitation is not effective in (de)populating $v=3$ because the densities and temperatures are too low. Thus, from the amount of absorption we can infer the ultraviolet flux (Federman et al. 1995).

The measurements of vibrationally excited $\mathrm{H}_{2}$ toward $\rho$ $\mathrm{Oph} \mathrm{A}$ are not easily modeled. The amount of $\mathrm{H}$ i along the line of sight is very large, which, together with the large amount of $\mathrm{H}_{2}$ in $v=3$, suggests that there is low-density gas close to the star. (Use of the revised $\mathrm{H}$ I column $\left[4.3 \times 10^{21}\right.$ $\mathrm{cm}^{-2}$ ] of Diplas \& Savage 1994 in the analysis does not remove the difficulties encountered here.) On the other hand, the $\mathrm{CO}$ column density is quite high, and $T_{10}\left(\mathrm{H}_{2}\right)$ is only $46 \mathrm{~K}$ (Savage et al. 1977), indicating the presence of denser, lower temperature gas along the line of sight. Rotational excitation in $\mathrm{C}_{2}$ is consistent with a total density of a few hundred $\mathrm{cm}^{-3}$, although the quality of the data is not very high (see Fig. 6). [The total density, $n_{\text {tot }}=n(\mathrm{H} \mathrm{I})+2 n\left(\mathrm{H}_{2}\right)>n_{c}$, is the parameter of interest in chemical modeling. For our sight lines, $n_{\text {tot }}$ is about $1.5 n_{c}$.]

The results from a set of models for $\rho$ Oph A appear in Table 7, where the first five entries are the input parameters for the models: the enhancement factor for the UV flux $\left(I_{\mathrm{UV}}\right), n_{\mathrm{tot}}$, the gas temperature $(T)$, the polytropic index, 
TABLE 7

Modeling Results for Vibrationally Excited $\mathrm{H}_{2}$ For $\rho$ OpH A

\begin{tabular}{|c|c|c|c|c|c|c|}
\hline \multirow[b]{2}{*}{ Parameter } & \multicolumn{5}{|c|}{ Model } & \multirow[b]{2}{*}{ ObSERVEd } \\
\hline & 1 & 2 & 3 & 4 & 5 & \\
\hline \multicolumn{7}{|c|}{ Model Parameters } \\
\hline$I_{\mathrm{UV}}$ & 4.0 & 10.0 & 10.0 & 10.0 & 8.0 & $\ldots$ \\
\hline$n_{\text {tot }}\left(\mathrm{cm}^{-3}\right)$ & 300.0 & 300.0 & 300.0 & $300.0^{\mathrm{a}}$ & $300.0^{\mathrm{a}}$ & $\ldots$ \\
\hline$T(\mathrm{~K})$ & 45.0 & 100.0 & 100.0 & $100.0^{\mathrm{a}}$ & $45.0^{\mathrm{a}}$ & $\ldots$ \\
\hline Index................................... & -1.00 & -1.00 & -1.00 & -1.20 & -1.18 & $\ldots$ \\
\hline Formation model .................... & $\mathrm{ST}^{\mathrm{b}}$ & ST & $\mathrm{W}^{\mathrm{c}}$ & ST & ST & $\cdots$ \\
\hline \multicolumn{7}{|c|}{$\begin{array}{l}\text { Column Densities } \\
\qquad\left(\mathrm{cm}^{-2}\right)\end{array}$} \\
\hline $\mathrm{H} \ldots \ldots \ldots \ldots$ & 4.8E20 & $6.6 \mathrm{E} 20$ & $6.6 \mathrm{E} 20$ & $1.0 \mathrm{E} 21$ & $1.1 \mathrm{E} 21$ & $6.5 \mathrm{E} 21$ \\
\hline . & 3.7E20 & 3.7E20 & 3.7E20 & 3.7E20 & $3.7 \mathrm{E} 20$ & 3.7E20 \\
\hline \multicolumn{7}{|l|}{$\mathrm{H}_{2} v=0$} \\
\hline$J=0 \ldots \ldots \ldots \ldots \ldots \ldots \ldots \ldots \ldots \ldots \ldots \ldots$ & $3.1 \mathrm{E} 20$ & $1.4 \mathrm{E} 20$ & 1.4E20 & 1.2E20 & $2.1 \mathrm{E} 20$ & $(3.0 \pm 1.0) \mathrm{E} 20$ \\
\hline$J=1$ & $6.4 \mathrm{E} 19$ & 2.3E20 & 2.3E20 & $2.5 \mathrm{E} 20$ & $1.6 \mathrm{E} 20$ & $(7.1 \pm 3.0) \mathrm{E} 19$ \\
\hline$J=2 \ldots \ldots$ & $1.7 \mathrm{E} 17$ & 2.7E18 & 2.7E18 & 4.9E18 & $1.6 \mathrm{E} 18$ & $\ldots$ \\
\hline$J=3 \ldots \ldots \ldots \ldots \ldots \ldots \ldots$ & $1.1 \mathrm{E} 16$ & $5.0 \mathrm{E} 16$ & $5.0 \mathrm{E} 16$ & $1.7 \mathrm{E} 17$ & 7.9E16 & $\ldots$ \\
\hline$J=4$ & $1.5 \mathrm{E} 15$ & $2.1 \mathrm{E} 15$ & $2.3 \mathrm{E} 15$ & $2.3 \mathrm{E} 15$ & $1.8 \mathrm{E} 15$ & $\ldots$ \\
\hline$J=5 \ldots \ldots \ldots \ldots \ldots \ldots \ldots \ldots \ldots \ldots \ldots \ldots \ldots$ & $2.9 \mathrm{E} 14$ & $7.0 \mathrm{E} 14$ & $7.2 \mathrm{E} 14$ & $7.7 \mathrm{E} 14$ & $5.2 \mathrm{E} 14$ & $\ldots$ \\
\hline \multicolumn{7}{|l|}{$\mathrm{H}_{2} v=3:$} \\
\hline$J=0 \ldots \ldots \ldots \ldots \ldots \ldots \ldots$ & $5.4 \mathrm{E} 11$ & 7.0E11 & 7.0E11 & $6.8 \mathrm{E} 11$ & 5.6E11 & $(8.2 \pm 2.0) \mathrm{E} 11$ \\
\hline$J=1$ & 7.4E11 & 2.3E12 & 2.3E12 & $2.6 \mathrm{E} 12$ & $1.6 \mathrm{E} 12$ & $(2.0 \pm 0.5) \mathrm{E} 12$ \\
\hline$J=2 \ldots \ldots \ldots \ldots \ldots \ldots \ldots \ldots \ldots \ldots$ & $1.3 \mathrm{E} 12$ & $1.7 \mathrm{E} 12$ & $1.7 \mathrm{E} 12$ & $1.7 \mathrm{E} 12$ & $1.4 \mathrm{E} 12$ & $(1.7 \pm 0.4) \mathrm{E} 12$ \\
\hline$J=3 \ldots \ldots \ldots \ldots \ldots \ldots \ldots$ & $6.4 \mathrm{E} 11$ & $2.0 \mathrm{E} 12$ & $1.9 \mathrm{E} 12$ & $2.2 \mathrm{E} 12$ & $1.4 \mathrm{E} 12$ & $(2.7 \pm 0.5) \mathrm{E} 12$ \\
\hline
\end{tabular}

NotE.-All models have the standard extinction curve with grain model 2.

a Temperature and density gradient: values refer to center of cloud.

b ST: statistical distribution of $1.5 \mathrm{eV}$ over all levels upon formation.

c W: Wagenblast 1992: fraction 0.73 in $v=5, J=9$ and 0.27 in $J=10$.

and the means of populating the levels during $\mathrm{H}_{2}$ formation. All our models are based on an adopted $b$-value of $1.0 \mathrm{~km}$ $\mathrm{s}^{-1}$. The initial model had constant $n_{\text {tot }}$ and $T$ with $n_{\mathrm{tot}}=300 \mathrm{~cm}^{-3}, T=45 \mathrm{~K}$, and $I_{\mathrm{UV}}=4$. This model reproduces the $\mathrm{H}_{2} v=0$ observations quite well, but it fails to reproduce those for $\mathrm{H}_{\mathrm{I}}$ and $\mathrm{H}_{2} v=3$. Increasing the radiation field to $I_{\mathrm{UV}}=10$ brings the column density for $\mathrm{H}_{2}$ in $v=3, J=0$ into agreement with observations, but this fails for the higher rotational levels. The $\mathrm{H}_{2}$ levels $v=3, J=1-3$ clearly indicate a much higher rotational temperature, between 100 and $500 \mathrm{~K}$; the second model shows the effects of increasing the temperature to $100 \mathrm{~K}$. This improves the comparison with the rotational levels in $v=3$, but now the column densities for $v=0$ are not consistent with observations. An attempt based on polytropic models with a temperature gradient from about $400 \mathrm{~K}$ at the edge to 45 or 100 $\mathrm{K}$ in the center still could not reproduce all the data. Given the limited set of data, especially the lack of data for the high-lying rotational levels in $v=0$, we can only state that there seem to be at least two components along the line of sight. A warm component of low-density gas is exposed to a radiation field that is enhanced by a factor of $\sim 10$ over the standard radiation field (Draine 1978); presumably, this gas is located close to the B2 V star, about 0.2 pc away. As noted in our work on $\zeta$ Oph (Federman et al. 1995), the current models do not include line overlap, which can result in an overestimate of the pumping rate by up to a factor of 2 . Thus, the $I_{\mathrm{UV}}$ inferred here for this component should be regarded as a lower limit. The second component, containing most of the $\mathrm{H}_{2} v=0$, as well as $\mathrm{CO}$ and $\mathrm{C}_{2}$, must be much colder and denser. This picture suggests that we are observing a photodissociation region in the reflection nebula associated with $\rho$ Oph via absorption lines. The two components may correspond to the dominant components in K I (Welty \& Hobbs 2001) and the two seen in CH spectra (Crane et al. 1995). Both $\mathrm{K} \mathrm{I}$ and $\mathrm{CH}$ probe material over a larger range in density than either $\mathrm{CO}$ or $\mathrm{C}_{2}$.

The direction to $\chi$ Oph was modeled by van Dishoeck \& Black (1986); their best model (G) yielded predictions for the amount of $\mathrm{H}_{2}$ in $v=3$ that exceed our upper limits by factors of $2-4$ or so (see Table 8). Therefore, the value for $I_{\mathrm{UV}}$ used in the calculations must be reduced accordingly, from which we infer that the strength of the ultraviolet field is comparable to the average interstellar field ( $I_{\mathrm{UV}}$ of $\left.1-2\right)$.

TABLE 8

Modeling Results for Vibrationally Excited $\mathrm{H}_{2}$ FOR $\chi$ OpH

\begin{tabular}{ccccc}
\hline \hline \multirow{2}{*}{ SPECIES } & \multicolumn{3}{c}{ ModEL } \\
\cline { 2 - 4 } & $1^{\mathrm{a}}$ & $2^{\mathrm{b}}$ & $3^{\mathrm{c}}$ & OBSERVED \\
\hline $\mathrm{H}_{2} v=3:$ & & & & \\
$J=0 \ldots \ldots \ldots \ldots$. & $1.0 \mathrm{E} 12$ & $1.6 \mathrm{E} 11$ & $3.0 \mathrm{E} 11$ & $\ldots$ \\
$J=1 \ldots \ldots \ldots \ldots \ldots$ & $1.4 \mathrm{E} 12$ & $2.2 \mathrm{E} 11$ & $4.2 \mathrm{E} 11$ & $\ldots$ \\
$J=2 \ldots \ldots \ldots \ldots \ldots$. & $2.4 \mathrm{E} 12$ & $3.8 \mathrm{E} 11$ & $7.1 \mathrm{E} 11$ & $\leq 4.8 \mathrm{E} 11$ \\
$J=3 \ldots \ldots \ldots \ldots .$. & $1.2 \mathrm{E} 12$ & $1.9 \mathrm{E} 11$ & $3.5 \mathrm{E} 11$ & $\leq 8.9 \mathrm{E} 11$ \\
\hline
\end{tabular}

a $\chi$ Oph model G: $T=45 \mathrm{~K}, I_{\mathrm{UV}}=9, n_{\mathrm{tot}}=300 \mathrm{~cm}^{-3}$; van Dishoeck \& Black 1986.

${ }^{\mathrm{b}}$ Model $\mathrm{G}$ with $I_{\mathrm{UV}}=1$.

c Model G with $I_{\mathrm{UV}}=2$. 
As shown in Table 8, new calculations with reduced field strengths confirm this supposition. The results of the simplified analysis of Federman et al. (1994b) now agree better with the refined models described here.

\subsection{CO Fractionation}

With the addition of the present results for $\rho$ Oph A and $\chi$ Oph, there are three sight lines in Sco OB2 with accurately determined values for the $N\left({ }^{12} \mathrm{CO}\right) / N\left({ }^{13} \mathrm{CO}\right)$ ratio. We have $125 \pm 23$ for $\rho$ Oph A, $117 \pm 35$ for $\chi$ Oph, and $167 \pm 15$ for $\zeta$ Oph (the latter from Lambert et al. 1994). The amount of fractionation is comparable in the gas toward $\rho \mathrm{Oph} \mathrm{A}$ and $\chi \mathrm{Oph}$, and it is larger toward $\zeta \mathrm{Oph}$. These ratios are greater than the ambient ${ }^{12} \mathrm{C} /{ }^{13} \mathrm{C}$ ratio of about 65; self-shielding of the more abundant isotopomer clearly causes the observed fractionation. The results can be understood in a qualitative way through the realization that larger column densities increase the effects of self-shielding, while larger fluxes of ultraviolet radiation increase the photodissociation rate. In other words, the "reduced column density" $N\left({ }^{12} \mathrm{CO}\right) / I_{\mathrm{UV}}$ is a measure of the amount of fractionation. Taking $N\left({ }^{12} \mathrm{CO}\right)$ from Table 5 and $I_{\mathrm{UV}}$ from the previous section, along with the results for $\zeta$ Oph from Lambert et al. (1994), $N\left({ }^{12} \mathrm{CO}\right)=2.5 \times 10^{15} \mathrm{~cm}^{-2}$, and Federman et al. (1995), $I_{\mathrm{UV}}=1-2$, we obtain values of $N\left({ }^{12} \mathrm{CO}\right) / I_{\mathrm{UV}}$ of about $1.9 \times 10^{14},(1.9-3.8) \times 10^{14}$, and $(1.3-2.5) \times 10^{15} \mathrm{~cm}^{-2}$ for $\rho$ Oph A, $\chi \mathrm{Oph}$, and $\zeta \mathrm{Oph}$, respectively. The reduced column density follows the trend seen in the observed amount of fractionation. For the directions considered here, selective isotopic photodissociation is the process controlling the relative abundances of the ${ }^{12} \mathrm{CO}$ and ${ }^{13} \mathrm{CO}$ isotopomers; the present results on $\mathrm{C}^{18} \mathrm{O}$ are not precise enough for definitive statements.

A more quantitative means of comparison involves analysis based on equation (15) in Lambert et al. (1994):

$$
\begin{aligned}
F_{13}= & \frac{\Gamma_{13}}{\Gamma_{12}}\left[1+\frac{3.2 \times 10^{-12}}{\Gamma_{13}}\left(\frac{n_{\mathrm{tot}}}{200}\right)\left(\frac{\delta_{\mathrm{C}}}{0.1}\right)\right] \\
& \times\left[1+\frac{6.4 \times 10^{-12}}{\Gamma_{12}}\left(\frac{n_{\mathrm{tot}}}{200}\right)\left(\frac{\delta_{\mathrm{C}}}{0.1}\right)\right]^{-1}
\end{aligned}
$$

In this expression, the amount of fractionation, $F_{13}$, is $\left[n\left({ }^{12} \mathrm{CO}\right) / n\left({ }^{13} \mathrm{CO}\right)\right] /\left[n\left({ }^{12} \mathrm{C}\right) / n\left({ }^{13} \mathrm{C}\right)\right]$ and the photodissociation rate for isotopomer $i$ is $\Gamma_{i}$. The numerical coefficients in square brackets are based on the rate constants for isotopic charge exchange, while $\delta_{\mathrm{C}}$ represents the elemental carbon abundance relative to the value for the Sun. A factor of 0.4 more closely matches the interstellar measurements of Cardelli et al. (1993b). The photodissociation rates come from the calculations of shielding functions by van Dishoeck \& Black (1988) - see their Table 5, which includes the effects of $\mathrm{CO}$ self-shielding and mutual shielding from $\mathrm{H}_{2}$. The necessary columns of $\mathrm{H}_{2}$ are taken from Savage et al. (1977). The amount of grain attenuation is based on model 2 described by van Dishoeck \& Black (1986). For $\rho$ Oph A and $\chi$ Oph, we multiplied the grain optical depth by 0.7 to accommodate larger than average values for the ratio of total to selective extinction (Federman et al. 1994b). We approximate local densities $n(\mathrm{X})$ with column densities. We considered $I_{\mathrm{UV}}=1$ toward $\chi$ Oph and $\zeta$ Oph and $I_{\mathrm{UV}}=10$ toward $\rho$ Oph A. For $n_{\text {tot }}$, we adopted $300 \mathrm{~cm}^{-3}$ for the gas toward $\rho \mathrm{Oph} \mathrm{A}$ and $\chi \mathrm{Oph}$, as inferred above, and $200 \mathrm{~cm}^{-3}$ for the gas toward $\zeta \mathrm{Oph}$
(Lambert et al. 1994). Finally, since van Dishoeck \& Black (1988) considered a slab geometry, the column densities used here are one-half the measured ones.

With the use of an ambient isotope ratio of 65 for carbon, our measurements indicate values for $F_{13}$ of $1.9 \pm 0.4$, $1.8 \pm 0.6$, and $2.6 \pm 0.3$ for the directions $\rho$ Oph A, $\chi \mathrm{Oph}$, and $\zeta$ Oph. The uncertainties in the observed values are based on the uncertainties in the ${ }^{12} \mathrm{CO} /{ }^{13} \mathrm{CO}$ ratios and an assumed $10 \%$ uncertainty in the ${ }^{12} \mathrm{C} /{ }^{13} \mathrm{C}$ ratio, taken in quadrature. The quantities in square brackets are approximately 1 when $n_{\text {tot }}$ of a few hundred $\mathrm{cm}^{-3}$ is adopted, as found from $\mathrm{C}_{\mathrm{I}}$ and $\mathrm{C}_{2}$ excitation. The main exceptions are the calculations for $\chi \mathrm{Oph}$ and $\zeta \mathrm{Oph}$, where the bracketed term involving $\Gamma_{12}$ is about 2.0. In other words, this reenforces the notion that the three sight lines are controlled by photodissociation. We find $F_{13}$ to be $1.8,1.0$, and 1.2 for the respective sight lines. Considering the simplifications made (e.g., column for local density), the accuracy of the observational results $(10 \%-30 \%)$, and the results using Table 5 of van Dishoeck \& Black (1988; 20\%-30\%), the agreement between calculations and observations is good. The correspondence for $\chi \mathrm{Oph}$ and $\zeta$ Oph could be improved by combined changes in $I_{\mathrm{UV}}$ and $n_{\mathrm{tot}}$ of about a factor of 4; this would be accomplished by increasing $I_{\mathrm{UV}}$ and decreasing $n_{\text {tot }}$. It is satisfying that our simple analyses utilizing observational input are able to reproduce the enhanced ${ }^{12} \mathrm{CO} /{ }^{13} \mathrm{CO}$ ratios seen by us. A more detailed modeling effort is beyond the scope of this paper.

\section{DISCUSSION}

\section{1. $\mathrm{H}_{2}$ Excitation}

When the populations of vibrationally excited $\mathrm{H}_{2}$ are controlled by optical pumping via absorption of UV photons, the ortho (odd $J$ ) to para (even $J$ ) ratio in excited states differs from the ratio for the vibrational ground state (Sternberg \& Neufeld 1999). While their work focuses on warm photodissociation regions, where the ortho-to-para ratio in the ground state is the thermal value, the same ideas apply to cool gas where the population in $J=0$ is large. The effect is clearly seen in our data for $\rho$ Oph A and the data of Federman et al. (1995) for $\zeta$ Oph. The respective ortho-topara ratios for the $v=3$ state toward the two stars are $2.7 \pm 0.9$ and $4.1 \pm 2.2$, and the corresponding ratios for the ground state are $0.7 \pm 0.3$ and $1.2 \pm 0.4$. For the $v=3$ state, we summed over all possible rotational levels, including statistical weights, but only considered the $J=0$ and 1 levels of the ground state because they have much larger columns than higher lying levels.

The difference in ortho-to-para ratio between vibrational levels arises from enhanced optical depth in lines from $J=0$ of the ground state. Since optical pumping populates the rotational levels of vibrationally excited states via a twostep process, the change in rotational quantum number is 0 or 2. The larger optical depth of lines originating from $J=0$, the most populous (para) level, reduces the abundance of para rotational levels in $v=3$. This leads to a larger ortho-to-para ratio for $v=3$.

Excitation of $\mathrm{H}_{2}$ also occurs during its formation on grains. During formation, about $4.4 \mathrm{eV}$ of binding energy is distributed among substrate excitation, molecular excitation, and translational energy. The partition among the channels is poorly understood. For instance, it is not clear whether the 
nascent molecules are vibrationally hot and rotationally cold - high $v$ and low $J$ (see Duley \& Williams 1993) - or the opposite (Hunter \& Watson 1978; Wagenblast 1992). More recent experimental (Gough et al. 1996) and theoretical (Kim, Ree, \& Shin 1999) work on $\mathrm{H}_{2}$ excitation during formation on carbonaceous surfaces suggests that most of the energy goes into vibrational motion. Internal excitation of $\mathrm{H}_{2}$ could be discerned from observations of high-lying rotational levels in the ground state and of vibrationally excited $\mathrm{H}_{2}$. Since high-lying rotational levels in the ground state are needed to assess the importance of the predicted distributions (Federman et al. 1995) but such data do not exist for $\rho$ Oph A, more definitive statements cannot be made at this time. The large number of rovibrational levels probed by observations of HD 37903 (Meyer et al. 2001) may provide the answer to this important question.

In our studies of molecular chemistry in diffuse clouds (e.g., van Dishoeck \& Black 1989; Federman et al. 1997a; Knauth et al. 2001), we have begun to appreciate the importance of using the extinction curve for the sight line in the analysis. As for the two directions examined here, the extinction curve for both stars is lower than "typical" at the shortest wavelengths (Fitzpatrick \& Massa 1990; Snow, Allen, \& Polidan 1990; Green et al. 1992). In the present work, we did not attempt to model more than $\mathrm{H}_{2}$ in any detail. Under these circumstances, different extinction curves could be incorporated into the scaling term $I_{\mathrm{UV}}$. It is also worth mentioning that calculations adopting bidirectional radiation fields, like those of Lyu et al. (1994), with one-half the flux incident on each side of the cloud yield results similar to the ones presented here.

\subsection{CO Fractionation}

There are now three sight lines, all in Sco-Oph, where ${ }^{12} \mathrm{CO}$ is enhanced relative to other isotopomers by approximately a factor of 2 over the ambient ${ }^{12} \mathrm{C} /{ }^{13} \mathrm{C}$ ratio. We are confident in our results for a number of reasons. First, other analyses that yielded smaller relative amounts of ${ }^{12} \mathrm{CO}$ from the $\mathrm{CO}$ data toward $\zeta$ Oph were based either on a set of $f$ values now known to be inaccurate (Lyu et al. 1994) or on data of the same bands for ${ }^{12} \mathrm{CO}$ and ${ }^{13} \mathrm{CO}$ (Levshakov \& Kegel 1994), which are prone to optical depth corrections in the more abundant isotopomer. The former point is addressed in more detail below. Second, our synthesis code does not always give results indicating enhanced amounts of ${ }^{12} \mathrm{CO}$. In a paper on photodissociation regions toward sources of reflection nebulae (Knauth et al. 2001), our code used for profile synthesis confirmed the lower ${ }^{12} \mathrm{CO} /{ }^{13} \mathrm{CO}$ ratio ( 40) seen toward 20 Aql (Hanson, Snow, \& Black 1992) from an independent extraction of $I U E$ spectra.

An especially critical one is the third reason. New measurements of $f$-values for Rydberg transitions of $\mathrm{CO}$ are larger than once thought (Federman et al. 2001). (These are the $f$-values giving excellent fits to the $\mathrm{CO}$ bands seen in Copernicus spectra, upon adoption of the parameters derived from the $A-X$ bands, as discussed in $\S 3$ and in Federman \& Lambert 2002.) Although only a few transitions of importance in $\mathrm{CO}$ photodissociation have been reexamined to date, in all cases the $f$-values for Rydberg transitions are about a factor of 2 larger than those compiled by Eidelsberg et al. (1991) from the measurements of Letzelter et al. (1987). This may explain why ab initio modeling efforts, such as those of van Dishoeck \& Black (1986,
1988), Kopp et al. (1996), and Warin et al. (1996), are unable to produce ${ }^{12} \mathrm{CO} /{ }^{13} \mathrm{CO}$ ratios much in excess of the ambient ${ }^{12} \mathrm{C} /{ }^{13} \mathrm{C}$ ratio. Use of a larger $f$-value would increase the optical depth of dissociating transitions, which in turn leads to more self-shielding for the more abundant isotopomer. Moreover, larger optical depths in lines leading to $\mathrm{CO}$ dissociation would improve the correspondence between model predictions and submillimeter-wave observations of dense photodissociation regions (Hollenbach \& Tielens 1999). Further work is needed to derive accurate $f$-values for all transitions involved in $\mathrm{CO}$ photodissociation.

More self-shielding for ${ }^{12} \mathrm{CO}$ would also provide a better correspondence in the $\mathrm{CO}$ abundance between model and observations. For instance, the theoretical predictions of van Dishoeck \& Black (1986) are too small by factors of a few, even after accounting for the smaller values for $I_{\mathrm{UV}}$ derived from vibrationally excited $\mathrm{H}_{2}$. An enhancement in $\mathrm{CO}$ production would help alleviate the problem as well. A key reaction is $\mathrm{C}^{+}+\mathrm{OH} \rightarrow \mathrm{CO}^{+}+\mathrm{H}$. Dubernet, Gargaud, $\&$ McCarroll (1992) performed quantal calculations on this reaction and obtained a rate constant of about $5 \times 10^{-9} \mathrm{~cm}^{3}$ $\mathrm{s}^{-1}$, which is substantially larger than the estimate given in Prasad \& Huntress (1980) and is about a factor of 2 larger than the value given by Federman \& Huntress (1989) based on average dipole orientation theory.

While a self-consistent picture seems to be emerging, we briefly discuss the apparent contradictory results of Lyu et al. (1994) and Liszt \& Lucas (1998). It is our contention that the differences between Lyu et al. and our earlier work (Sheffer et al. 1992; Lambert et al. 1994) for $\zeta$ Oph lie in the adopted $f$-values. As noted in the Introduction, Lambert et al. found a more satisfactory curve of growth with the $f$-values of Chan et al. (1993). All agree that the column density of ${ }^{12} \mathrm{CO}$ is best determined by the $11-0$ and $12-0$ bands, which are essentially optically thin. For these bands, the $f$-values of Eidelsberg et al. (1992) are 50\% larger than those of Chan et al. With their preferred curve of growth, Lyu et al. infer $N\left({ }^{12} \mathrm{CO}\right)=1.8 \times 10^{15} \mathrm{~cm}^{-2}$; this value becomes $2.7 \times 10^{15} \mathrm{~cm}^{-2}$ when the $f$-values of Chan et al. are used, much more in line with the results of Lambert et al. $\left(2.5 \times 10^{15} \mathrm{~cm}^{-2}\right)$. We also point out that the two sets of results for the 5-0 band of ${ }^{13} \mathrm{CO}$ agree nicely. There is another way to check the ${ }^{12} \mathrm{CO} /{ }^{13} \mathrm{CO}$ ratios: the ratio of $f$-values for ${ }^{13} \mathrm{CO}$ and ${ }^{12} \mathrm{CO}$ bands with the same $W_{\lambda}$. Lambert et al. provided a comparison in their Table 5, which indicates a ratio greater than 125 . The same comparison can be made with our data displayed in Table 2. For both $\rho$ Oph A and $\chi$ Oph, the $W_{\lambda}$ for the $11-0$ band of ${ }^{12} \mathrm{CO}$ is the same as that for the $4-0$ band of ${ }^{13} \mathrm{CO}$. The ratio of $f$-values is 134 , consistent with our isotopomeric ratios.

Finally, we comment on the work of Liszt \& Lucas (1998), where the ${ }^{12} \mathrm{CO} /{ }^{13} \mathrm{CO}$ ratio decreases with increasing $N\left({ }^{12} \mathrm{CO}\right)$ for column densities sampled by our UV measurements. First, our results specifically concern the clouds in Sco-Oph; other clouds, like that toward $20 \mathrm{Aql}$, have much smaller ratios (Hanson et al. 1992; Knauth et al. 2001). Second, there are no direct measures of extinction or $\mathrm{C}^{+}$abundance for their extragalactic sight lines. Enhancements in ${ }^{13} \mathrm{CO}$ relative to ${ }^{12} \mathrm{CO}$ require a significant flux of $\mathrm{UV}$ radiation to produce the $\mathrm{C}^{+}$needed for isotopic charge exchange (but not too large to heat the gas via photoelectrons coming off grains). A more accurate determination of the extinction would also help clarify why complex molecules such as HCN (e.g., Lucas \& Liszt 1994) are detected, 
when standard chemical models of diffuse molecular gas (e.g., van Dishoeck \& Black 1986) suggest very low abundances.

In summary, ultraviolet observations of $\mathrm{CO}$ and its isotopic variants toward $\rho$ Oph A and $\chi$ Oph were analyzed in conjunction with measurements on absorption from $\mathrm{C}$ I and vibrationally excited $\mathrm{H}_{2}$. Significant enhancements in the amount of ${ }^{12} \mathrm{CO}$ relative to the other isotopomers were found. The amount of $\mathrm{H}_{2}$ in $v=3$ toward $\rho \mathrm{Oph} \mathrm{A}$ indicates that an ultraviolet flux about 10 times the average interstellar flux permeates this direction; for the $\chi$ Oph sight line, the flux cannot be greater than twice the average interstellar value. Analysis of $\mathrm{C}_{\mathrm{I}}$ (and $\mathrm{C}_{2}$ ) excitation reveals modest densities for the material seen in absorption. Resonant scattering of emission lines from nearby molecular clouds controls the distribution of $\mathrm{CO}$ rotational levels, thereby limiting the usefulness of $\mathrm{CO}$ excitation for inferring density. Simple arguments based on the processes involved in selective isotopic photodissociation are able to reproduce the observed ${ }^{12} \mathrm{CO} /{ }^{13} \mathrm{CO}$ ratios. Incorporation of an improved set of $f$-values for dissociating transitions in largescale models will likely lead to better agreement with our observations.

The archive of Copernicus data developed by George Sonneborn and available at the Multiwavelength Archive at the Space Telescope Science Institute was used in this research. Support for this work was provided by NASA through grant GO-5389.02-93A from the Space Telescope Science Institute, which is operated by the Association of Universities for Research in Astronomy, Inc., under NASA contract NAS5-26555 and through Long Term Space Astrophysics grant NAG5-4957 to the University of Toledo. We acknowledge the helpful suggestions of an anonymous referee.

\section{APPENDIX}

\section{ABUNDANCES OF HEAVY ELEMENTS}

In addition to lines of CO, $\mathrm{H}_{2}, \mathrm{C}$ I, S I, Co II, and Ni II reported here and in Zsargó et al. (1997), Mullman et al. (1998), and Zsargó \& Federman (1998), our spectra of $\rho$ Oph A and $\chi$ Oph reveal absorption from Ga II, As II, and Sn II. The results are given in Table 9, where $W_{\lambda}$ and $N(\mathrm{X})$ are listed. The sources of $f$-values used in deriving column densities are Morton (1991) for Ga II, Cardelli et al. (1993a) for As II, and Schectman et al. (2000) for Sn II. A curve of growth with a $b$-value of $2.5 \mathrm{~km} \mathrm{~s}^{-1}$ was used; such a $b$-value is common for the dominant ion (Savage, Cardelli, \& Sofia 1992). Two components, separated by about $10 \mathrm{~km} \mathrm{~s}^{-1}$, are discerned in the spectra of $\chi$ Oph. These are the major complexes found in Na I D (Welty, Hobbs, \& Kulkarni 1994). Furthermore, knowledge of the total proton column density yields an elemental abundance. We adopted a combination of the $\mathrm{H}_{2}$ data of Savage et al. (1977) and $\mathrm{H}$ I data of Diplas \& Savage (1994) for $\rho \mathrm{Oph} \mathrm{A}, N_{\mathrm{H}}=5.0 \times 10^{21} \mathrm{~cm}^{-2}$, and the results of Bohlin, Savage, \& Drake (1978) for $\chi \mathrm{Oph}, 2.26 \times 10^{21} \mathrm{~cm}^{-2}$. The fractional abundances $\left(\approx 10^{-10}\right)$ are similar to those seen toward $\zeta$ Oph (Cardelli, Savage, \& Ebbets 1991; Cardelli et al. 1993a) and in other $\mathrm{H}_{2}$-rich sight lines (Cardelli 1994 for Ga II; Sofia, Meyer, \& Cardelli 1999 for Sn II).

TABLE 9

Results For Ga II, As II, AND Sn II

\begin{tabular}{|c|c|c|c|c|c|c|}
\hline \multirow[b]{2}{*}{ SPECIES } & \multirow[b]{2}{*}{$\begin{array}{l}\text { LiNE } \\
(\AA)\end{array}$} & \multirow[b]{2}{*}{$f$-VALUE } & \multicolumn{2}{|c|}{$\rho$ ОРн A } & \multicolumn{2}{|c|}{$\chi \mathrm{OPH}$} \\
\hline & & & $\begin{array}{c}W_{\lambda} \\
(\mathrm{m} \AA)\end{array}$ & $\begin{array}{c}N(\mathrm{X}) \\
\left(\times 10^{11} \mathrm{~cm}^{-2}\right)\end{array}$ & $\begin{array}{c}W_{\lambda} \\
(\mathrm{m} \AA)\end{array}$ & $\begin{array}{c}N(\mathrm{X}) \\
\left(\times 10^{11} \mathrm{~cm}^{-2}\right)\end{array}$ \\
\hline 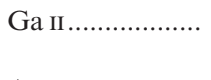 & 1414.40 & $1.80^{\mathrm{a}}$ & $\begin{array}{c}11.28 \pm 0.35 \\
\ldots\end{array}$ & $\begin{array}{c}4.38 \pm 0.17 \\
\ldots\end{array}$ & $\begin{array}{l}0.81 \pm 0.16 \\
5.41 \pm 0.16\end{array}$ & $\begin{array}{l}0.26 \pm 0.05^{\mathrm{b}} \\
1.87 \pm 0.06^{\mathrm{b}}\end{array}$ \\
\hline As II ..................... & 1263.77 & $0.32^{\mathrm{c}}$ & $1.23 \pm 0.35$ & $2.78 \pm 0.81$ & $0.67 \pm 0.24$ & $1.50 \pm 0.55$ \\
\hline 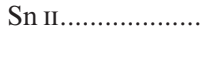 & 1400.44 & $1.04^{\mathrm{d}}$ & $\begin{array}{c}4.69 \pm 0.33 \\
\ldots\end{array}$ & $\begin{array}{c}2.82 \pm 0.22 \\
\ldots\end{array}$ & $\begin{array}{l}0.41 \pm 0.16 \\
3.02 \pm 0.16\end{array}$ & $\begin{array}{l}0.24 \pm 0.09^{\mathrm{b}} \\
1.76 \pm 0.10^{\mathrm{b}}\end{array}$ \\
\hline
\end{tabular}

\footnotetext{
${ }^{\text {a }}$ From Morton 1991.

b There are two velocity components, separated by approximately $10 \mathrm{~km} \mathrm{~s}^{-1}$.

${ }^{c}$ From Cardelli et al. 1993a.

d From Schectman et al. 2000.
} 
Auguson, G. C. \& Herbig, G. H. 1967, ApJ, 150, 729

Bally, J., \& Langer, W. D. 1982, ApJ, 255, 143 (erratum 261, 747)

Bembenek, Z. 1997, J. Mol. Spectrosc., 181, 136

Bensch, F., Pak, I., Wouterloot, J. G. A., Klapper, G., \& Winnewisser, G. 2001, ApJ, 562, L185

Bernes, C. 1979, A\&A, 73, 67

Black, J. H., \& Dalgarno, A. 1976, ApJ, 203, 132

Black, J. H., \& van Dishoeck, E. F. 1987, ApJ, 322, 412

Bohlin, R. C., Savage, B. D., \& Drake, J. F. 1978, ApJ, 224, 132

Cardelli, J. A. 1994, Science, 265, 209

Cardelli, J. A., \& Ebbets, D. C. 1994, in Calibrating Hubble Space Telescope, HST Calibration Workshop, ed. J. C. Blades \& A. J. Osmer (Baltimore: STScI), 322

Cardelli, J. A., Federman, S. R., Lambert, D. L., \& Theodosiou, C. E. 1993a, ApJ, 416, L41

Cardelli, J. A., Mathis, J. S., Ebbets, D. C., \& Savage, B. D. 1993b, ApJ, 402, L17

Cardelli, J. A., Savage, B. D., \& Ebbets, D. C. 1991, ApJ, 383, L23

Carrington, A., \& Ramsay, D. A. 1982, Phys. Scr., 25, 272

Centurion, M., Cassola, C., \& Vladilo, G. 1995, A\&A, 302, 243 (CCV95)

Centurion, M., \& Vladilo, G. 1991, A\&A, 251, 245

Chan, W. F., Cooper, G., \& Brion, C. E. 1993, Chem. Phys., 170, 123

Chu, Y.-H., \& Watson, W. D. 1983, ApJ, 267, 151

Crane, P., Hegyi, D. J., \& Lambert, D. L. 1991, ApJ, 378, 181

Crane, P., Lambert, D. L., \& Sheffer, Y. 1995, ApJS, 99, 107

Danks, A. C., \& Lambert, D. L. 1983, A\&A, 124, 188

de Geus, E. J., Bronfman, L., \& Thaddeus, P. 1990, A\&A, 231, 137

Diplas, A., \& Savage, B. D. 1994, ApJS, 93, 211

Draine, B. T. 1978, ApJS, 36, 595

Dubernet, M. L., Gargaud, M., \& McCarroll, R. 1992, A\&A, 259, 373

Duley, W. W., \& Williams, D. A. 1993, MNRAS, 260, 37

Eidelsberg, M., Benayoun, J. J., Viala, Y., \& Rostas, F. 1991, A\&AS, 90, 231

Eidelsberg, M., Jolly, A., Lemaire, J. L., Tchang-Brillet, W.-ÜL., Breton, J., \& Rostas, F. 1999, A\&A, 346, 705

Eidelsberg, M., \& Rostas, F. 2003, ApJS, 145, 89

Eidelsberg, M., Rostas, F., Breton, J., \& Thieblemont, B. 1992, J. Chem. Phys., 96, 5585

Erman, P., \& Iwamae, A. 1995, ApJ, 450, L31

Federman, S. R., Cardelli, J. A., Sheffer, Y., Lambert, D. L., \& Morton, D. C. 1994a, ApJ, 432, L139

Federman, S. R., Cardelli, J. A., van Dishoeck, E. F., Lambert, D. L., \& Black, J. H. 1995, ApJ, 445, 325

Federman, S. R., Fritts, M., Cheng, S., Menningen, K. L., Knauth, D. C., \& Fulk, K. 2001, ApJS, 134, 133

Federman, S. R., Glassgold, A. E., Jenkins, E. B., \& Shaya, E. J. 1980, ApJ, 242,545

Federman, S. R., \& Huntress, W. T., Jr. 1989, ApJ, 338, 140

Federman, S. R., Knauth, D. C., Lambert, D. L., \& Andersson, B.-G. 1997a, ApJ, 489, 758

Federman, S. R., \& Lambert, D. L. 2002, J. Electron Spectrosc. Relat. Phenomena, 123,161

Federman, S. R., Menningen, K. L., Lee, W., \& Stoll, J. B. 1997b, ApJ, 477, L61

Federman, S. R., Strom, C. J., Lambert, D. L., Cardelli, J. A., Smith, V. V. \& Joseph, C. L. 1994b, ApJ, 424, 772

Federman, S. R., Welty, D. E., \& Cardelli, J. A. 1997c, ApJ, 481, 795

Federman, S. R., \& Zsargó, J. 2001, ApJ, 555, 1020

Fitzpatrick, E. L., \& Massa, D. 1990, ApJS, 72, 163

Frisch, P. C. 1980, ApJ, 241, 697

Gough, S., Schermann, C., Pichou, F., Landau, M., Čadež, I., \& Hall, R. I. 1996, A\&A, 305, 687

Gredel, R. van Dishoeck, E. F \& Black, J. H. 1994, A\&A, 285, 300

Green, J. C., Snow, T. P., Jr., Cook, T. A., Cash, W. C., \& Poplawski, D. 1992, ApJ, 395, 289

Hanson, M. M., Snow, T. P., \& Black, J. H. 1992, ApJ, 392, 571

Hogerheijde, M. R., \& van der Tak, F. F. S. 2000, A\&A, 362, 697

Hollenbach, D. J., \& Tielens, A. G. G. M. 1999, Rev. Mod. Phys., 71, 173

Hunter, D. A., \& Watson, W. D. 1978, ApJ, 226, 477

Jenkins, E. B., Jura, M. \& Loewenstein, M. 1983, ApJ, 270, 88

Jolly, A., Lemaire, J. L., Belle-Oudry, D., Edwards, S., Malmasson, D., Vient, A., \& Rostas, F. 1997, J. Phys. B, 30, 4315
Kim, Y. H., Ree, J. \& Shin, H. K. 1999, Chem. Phys. Lett., 314, 1

Knauth, D. C., Federman, S. R., \& Lambert, D. L. 2003, ApJ, 586, 268

Knauth, D. C., Federman, S. R., Pan, K., Yan, M., \& Lambert, D. L. 2001, ApJS, 135, 201

Kopp, M., Gerin, M., Roueff, E. \& Le Bourlot, J. 1996, A\&A, 305, 558

Lambert, D. L., \& Danks, A. C. 1986, ApJ, 303, 401

Lambert, D. L., Sheffer, Y., \& Federman, S. R. 1995, ApJ, 438, 740

Lambert, D. L., Sheffer, Y., Gilliland, R. L., \& Federman, S. R. 1994, ApJ, 420,756

Langer, W. D., Glassgold, A. E., \& Wilson, R. W. 1987, ApJ, 322, 450

Langer, W. D., \& Penzias, A. A. 1993, ApJ, 408, 539

Langhoff, S. R., Bauschlicher, C. W., Jr., Rendell, A. P., \& Komornicki, A. 1990, J. Chem. Phys., 92, 3000

Lavendy, H., Robbe, J. M., Chambaud, G., Levy, R., \& Roueff, E. 1991, A\&A, 251, 365

Letzelter, C., Eidelsberg, M., Rostas, F., Breton, J., \& Thieblemont, B. 1987, Chem. Phys., 114, 273

Levshakov, S. A., \& Kegel, W. H. 1994, MNRAS, 271, 161

Liszt, H. S., \& Lucas, R. 1998, A\&A, 339, 561

Lucas, R. \& Liszt, H. S. 1994, A\&A, 282, L5

Lyu, C.-H., Smith, A. M. \& Bruhweiler, F. C. 1994, ApJ, 426, 254

Meyer, D. M., Lauroesch, J. T., Sofia, U. J., Draine, B. T., \& Bertoldi, F. 2001, ApJ, 553, L59

Morton, D. C. 1975, ApJ, 197, 85

1991, ApJS, 77, 119

Morton, D. C., \& Noreau, L. 1994, ApJS, 95, 301

Mullman, K. L., Lawler, J. E., Zsargó, J., \& Federman, S. R. 1998, ApJ, 500,1064

Penzias, A. A. 1981, ApJ, 249, 518

Phillips, T. R. 1994, MNRAS, 271, 827

Prasad, S. S., \& Huntress, W. T., Jr. 1980, ApJS, 43, 1

Robbe, J. M., Lavendy, H., Lemoine, D., \& Pouilly, B. 1992, A\&A, 256, 679

Rostas, F., Eidelsberg, M., Jolly, A., Lemaire, J. L., Le Floch, A., \& Rostas, J. 2000, J. Chem. Phys., 112, 4591

Savage, B. D. Cardelli, J. A., \& Sofia, U. J. 1992, ApJ, 401, 706

Savage, B. D., Drake, J. F., Budich, W., \& Bohlin, R. C. 1977, ApJ, 216, 291

Schectman, R. M., Cheng, S., Curtis, L. J., Federman, S. R., Fritts, M. C., \& Irving, R. E. 2000, ApJ, 542, 400

Sheffer, Y., Federman, S. R., \& Lambert, D. L. 2002, ApJ, 572, L95

Sheffer, Y., Federman, S. R., Lambert, D. L., \& Cardelli, J. A. 1992, ApJ, 397,482

Smith, A. M. \& Stecher, T. P. 1971, ApJ, 164, L43

Smith, P. L., Stark, G., Yoshino, K., \& Ito, K. 1994, ApJ, 431, L143

Snow, T. P., Jr., Allen, M. M., \& Polidan, R. S. 1990, ApJ, 359, L23

Snow, T. P., Jr., \& Jenkins, E. B. 1980, ApJ, 241, 161

Sofia, U. J., Meyer, D. M., \& Cardelli, J. A. 1999, ApJ, 522, L137

Stahl, O., \& Wilson, T. L. 1992, A\&A, 254, 327

Stahl, O., Wilson, T. L., Henkel, C., \& Appenzeller, I. 1989, A\&A, 221, 321

Stark, G., Lewis, B. R., Gibson, S. T., \& England, J. P. 1998, ApJ, 505, 452

Sternberg, A., \& Neufeld, D. A. 1999, ApJ, 516, 371

van Dishoeck, E. F., \& Black, J. H. 1982, ApJ, 258, 533

- 1986, ApJS, 62, 109

1988, ApJ, 334, 771

1989, ApJ 340, 273

van Dishoeck, E. F., \& de Zeeuw, T. 1984, MNRAS, 206, 383

Vladilo, G., Centurion, M. \& Cassola, C. 1993, A\&A, 273, 239

Wagenblast, R. 1992, MNRAS, 259, 155

Wannier, P., Penprase, B. E., \& Andersson, B.-G. 1997, ApJ, 487, L165

Warin, S., Benayoun, J. J., \& Viala, Y. P. 1996, A\&A, 308, 535

Watson, W. D., Anicich, V. G., \& Huntress, W. T., Jr. 1976, ApJ, 205, L165

Welty, D. E., \& Hobbs, L. M. 2001, ApJS, 133, 345

Welty, D. E., Hobbs, L. M., \& Kulkarni, V. P. 1994, ApJ, 436, 152

Wilson, T. L., \& Rood, R. 1994, ARA\&A, 32, 191

Zhong, Z. P., Feng, R. F., Xu, K. Z., Wu, S. L., Zhu, L. F., Zhang, X. J., Ji, Q., \& Shi, Q. C. 1997, Phys. Rev. A, 55, 1799

Zsargó, J., \& Federman, S. R. 1998, ApJ, 498, 256

. 2003, ApJ, 589, 319

Zsargó, J., Federman, S. R., \& Cardelli, J. A. 1997, ApJ, 484, 820 